CERE Working Paper, 2012:10

\title{
Two-step approach to Self-Selected Interval Data in Elicitation Surveys
}

Yuri Belyaev ${ }^{1}$ and Bengt Kriström ${ }^{1,2}$

${ }^{1}$ Department of Forest Economics

SLU

S-901 83 Umeå, Sweden

${ }^{2}$ CERE, Centre for Environmental and Resource Economics

SLU and Umeå University

S-901 83 Umeå, Sweden

The Centre for Environmental and Resource Economics (CERE) is an inter-disciplinary and inter-university research centre at the Umeå Campus: Umeå University and the Swedish University of Agricultural Sciences. The main objectives with the Centre are to tie together research groups at the different departments and universities; provide seminars and workshops within the field of environmental \& resource economics and management; and constitute a platform for a creative and strong research environment within the field.

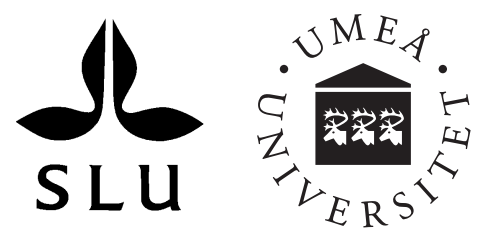

Department of Economics, Umeå University, S-901 87, Umeå, Sweden 


\title{
Two-step approach to Self-Selected Interval Data in Elicitation Surveys
}

\author{
Yuri Belyaev and Bengt Kriström
}

May 30, 2012 
TWO-STEP APPROACH TO SELF-SELECTED INTERVAL DATA IN ELICITATION SURVEYS ${ }^{1}$

\section{Yuri BELYAEV ${ }^{\mathrm{a}}$ AND BENGT KRISTRÖM ${ }^{\mathrm{b}}$}

We propose a novel two-step approach to elicitation in surveys and provide supporting statistical theory for the models suggested. The essential idea is to combine self-selected intervals in a first step and then employ brackets generated from the intervals in a second step. In this way we combine the advantages of selfselected intervals, mainly related to the fact that individuals often find it difficult to report a precise point-estimate of a quantity of interest, with the documented usefulness of brackets. Because the brackets are generated from the first sample we sidestep the thorny problem of the optimal design of brackets and additional assumptions on dependency between the self-selected intervals and their points of interest. Our set-up necessitates development of new statistical models. First, we propose a stopping rule for sampling in the first step. Second, Theorem 1 proves that the proposed non-parametric ML-estimator of the underlying distribution function is consistent. Third, a special recursion for quick estimation of the ML-

\footnotetext{
${ }^{1}$ Department of Forest Economics, 90183 Umeå, Sweden. The research presented in
} this paper was carried out as a part of the R\&D programme "Hydropower - Environmental impacts, mitigation measures and costs in regulated waters". It has been established and financed by Elforsk, the Swedish Energy Agency, the National Board of Fisheries and the Swedish Environmental Protection Agency. Partial funding from the "PlusMinus Program" (http://www.plusminus.slu.se) is also gratefully acknowledged. We thank the participants at the 15th Ulvön Conference on Environmental Economics, Ulvön, Sweden, June 17-19, 2008, 16th Annual Conference of the European Association for Environmental and Resource Economics, Göteborg, Sweden, June 25-28, 2008, IISA Conference Jan 4-8, 2010, Visakhapatnam, (Andhra U., Waltair), India, and seminar participants at the Department of Mathematics \& Mathematical Statistics, University of Umeå and Department of Forest Economics, SLU-Umeå, for useful input on earlier versions. Special thanks to Cecilia Håkansson for contributing the data and providing other useful input to the paper. The usual disclaimer applies.

aDepartment of Forest Economics SLU, $901 \quad 83$ Umeå, Sweden. yuri.belyaev@sekon.slu.se; http://www.sekon.slu.se

${ }^{\mathrm{b}}$ Centre for Environmental \& Resource Economics (CERE), 90183 Umeå, Sweden. Sweden. bengt.kristrom@sekon.slu.se; http://www. cere.se 
estimators is suggested. Theorem 2 shows that the accuracy of the estimator can be consistently estimated by resampling. Fourth, we have developed an Rpackage for efficient application of the method. We illustrate the approach using the problem of eliciting willingness-to-pay for a public good.

KEYwORDs: Interval data, Maximum Likelihood, Turnbull estimator, willingness-to-pay, quantitative elicitation, resampling..

\section{INTRODUCTION}

When asking quantitative survey-questions, there is an intuitively appealing advantage in letting the respondent choose an interval, rather than forcing him or her to state a point (or to choose one of a number of presented brackets). A point (or a given bracket) is , for all practical purposes, a special case of a self-selected interval. Several empirically observed phenomena provides additional motivation. One is that the respondent might not be completely certain about the answer. When asked about, say, the distance to the next city, we typically use a convenient round number or present an interval ("it is about 15-20 miles from here"). It seems natural that the elicitation mechanism should allow for such behavior. The individual might also refuse to answer the question because of uncertainty, see Belyaev and Kriström (2010, 2012), Håkansson (2007, 2008) and Manski and Molinari (2010) for evidence supporting this claim. Furthermore, because uncertainty may vary in complex ways across individuals, a freely chosen interval intuitively provides a better way of characterizing the underlying uncertainty, compared to brackets or open-ended questions.

Perhaps surprisingly, self-selected intervals are uncommon in the survey literature. One reason could be that the handling of such interval data is not part of the standard statistical literature. We propose to close some of this gap, by proposing a statistical toolbox for such interval-questions, complete with supporting statistical theory and publicly available software (written in R) Wenchao, Belyaev and Kriström (2012). 
The paper contains several new ideas and results. In survival analysis it is typically assumed that the censoring intervals are independent of the unobservable points of interest and that the intervals cover only some of the points, see Jammalamadaka and Mangalam (2003), Klein and Moeschberger (1997), Turnbull (1976). Our model does not put any restriction on the dependence between self-selected intervals and their associated (but unobservable) points; the model can be viewed as a generalization of the nonparametric ML-estimator for general censoring proposed by Turnbull (1976). However, the use of a single self-selected interval turns out to lead to a fundamental identifiability issue. To overcome this problem, we introduce a new two-step approach. We thus employ a second question in second sample, which we use to fine-grain the information from the first step. The second step depends on the first in a way that has not been explored before in the survey literature: the second step brackets are generated by self-selected intervals collected on the first step. We derive a sampling stopping rule which enables the analyst to formally address the problem of sample size in the first step. We couch our model within a contingent valuation experiment, in which the individual is asked about his willingness-to-pay (WTP) for a public good. This is a natural application of our ideas, because such questions typically involve unfamiliar goods (such as improvements in environmental quality) and a choice situation that individuals are typically not familiar with. While this is a natural application our approach has wide applicability.

The paper is structured as follows. In section 2 we describe basic assumptions and present the two-step sampling plan. In Section 3 we analyze intervals, stated by randomly sampled respondents, by using multinomial processes. Section 4 details the statistical model and the corresponding loglikelihood. Theorem 4.1 collects the properties of the proposed estimators and Theorem 4.2 gives properties of their accuracy. Section 5 provides a 
simulation experiment. Section 6 concludes. Proofs are in an Appendix.

\section{BASIC ASSUMPTIONS}

Surveys make up the life-blood of empirical research in the social sciences in general and in economics in particular. Employment surveys, investment surveys, inflation surveys are traditional examples, to which we can add the growing recent literature on the valuation of public goods. A key issue in any survey is the elicitation architecture, i.e. the way of elicitating information from the respondent. ${ }^{1}$ The choice is essentially between two types of survey questions, the open-ended and the closed-ended. ${ }^{2}$ Rather than eliciting a point estimate or select between given brackets, we first ask the individual to choose any interval of choice containing the point of interest.

Potential applications include, but are not limited to: recall situations ("How many days were you unemployed the first quarter of last year?", "What was your net income the previous year of taxation"?), projections ("What is your best forecast of the next year's interest rate?") or contingent valuation studies ("How much are you maximally willing to pay for the

\footnotetext{
${ }^{1}$ Perhaps the closest literature to our general approach is significant body of literature
} in psychology, statistics and survey research that provides approaches to elicit probability distributions, for a survey, see e.g. Garthwaite, Kadane and O'Hagan (2004). A compact survey of many issues in survey research, in particular regarding response errors and biases across formats is given in McFadden et al. (2005).

${ }^{2}$ Each of these can be further sub-divided into several categories. For example, closedended questions can be based on a Likert-scale (e.g. from "strongly disagree" to "strongly agree"), a multiple choice (e.g. 'circle one of the following alternatives'), an ordinal question (e.g. 'rank the following items from 1 to 5'), and a binary question (" are you willing to pay x USD for this public good" (yes, no)). There are also several variants of the openended questions, such as "How much are you willing to pay for this public good?" or "How did you make that choice?". The choice between the open and closed-ended questions is not straightforward, because they have advantages and disadvantages in different situations, see Fink (1985).

ectaart.cls ver. 2006/04/11 file: JASA_NOTBLIND.tex date: May 30, 2012 
suggested change?"). As Manski and Molinari (2010) points out intervals are more common in daily communication than we ordinarily think. Thus, weather reports and pilot communications include a form of implicit interval, e.g. when meteorologists report that the wind blows from the "north means that the wind direction lies in the interval [337.5., 22.5.]." This kind of rounding is also prevalent in many types of surveys; it is well-documented that individuals often round their answers to open-ended survey questions, see Rosch (1975), Schaeffer and Bradburn (1989), Huttenlocher, Hedges and Bradburn (2008), Hurd et al. (1998), Hobbs (2004), and van Exel et al. (2006).

The basic problem is that when a respondent is to report a point, he sometimes round it to describe a sentiment that really is an interval. Manski and Molinari (2010) present an approach to deal with such intervals, which is different from the one suggested here. The difference arises partly because we ask the respondent to select a point or an interval, not both. In addition, we take the view that the individual chooses a particular interval from an unobserved set of admissible intervals.

Another advantage with the self-selected intervals is that they arguably provide a richer picture of any underlying response uncertainty, compared to bracketing and some recent approaches to cater for respondent uncertainty in contingent valuation. The currently most popular elicitation approach in contingent valuation is a payment card containing several different costs for a public good, combined with a question about how certain the respondent feels about paying a certain cost (e.g. "definite yes", "probably yes", "probably no" and "definite no"). Recent analysis shows that including such uncertainty-assessments in the survey instrument may affect the estimate of valuations. For a review of this literature in this area see Broberg and Brännlund (2008). The intervals do not burden the respondent with the task of categorizing his uncertainty about a certain quantity and no issues arise 
as to how such categories should be represented in an econometric model. ${ }^{3}$

Our illustration provides an example from contingent valuation, a widely used approach to elicit individual's sentiment about non-priced goods or services (eg environmental quality). Our objective is to measure WTP (for an environmental improvement) and we will base our model on three basic assumptions:

Assumption 1 Each respondent might not be aware of the exact location of the true WTP-point. The respondents may freely choose intervals containing their true WTP-points. The ends of stated intervals may be rounded, e.g. to simple sums of coins or paper values of money.

Assumption 2 The true WTP-points are independent of question mode, i.e. the content of a question does not change the true WTP-point in the self-selected WTP-interval.

Assumption 3 The pairs of true WTP-points and the stated self-selected WTP-intervals, corresponding to different sampled individuals, are values of independent identically distributed (i.i.d.) random variables (r.v.s).

The first part of Assumption 1 has ample support in the contingent valuation literature. In the second part we naturally assume (but do not explicitly state) that the individual chooses an interval such that WTP does not exceed his or her income. We explicitly allow for rounding in the final part of Assumption 1. This is seldom, if ever, made explicit in the contingent valuation literature. Assumption 2 is important. From some perspectives, this assumption is very strong, given the evidence that exists on the differing results between types of valuation questions. L et us come back to it later.

\footnotetext{
${ }^{3}$ Each category of uncertainty can be considered in terms of a threshold-parameter in an ordered logit(probit) model. Assumptions are usually imposed on those parameters, an issue avoided here.
}

ectaart.cls ver. 2006/04/11 file: JASA_NOTBLIND.tex date: May 30, 2012 
Assumption 3 is, typically, innocuous, in that most contingent valuation applications are based on significant national samples.

\section{SAMPLING DESIGN}

We consider the following two-step plan of data collecting. On the first step randomly sampled individuals provide self-selected intervals that contain the true point. Let $n$, randomly sampled respondents, from a population $\mathfrak{P}$ of interest, have stated intervals $\mathbf{y}_{1}^{n}=\left\{\mathbf{y}_{1}, \ldots, \mathbf{y}_{n}\right\}, \mathbf{y}_{i}=\left(y_{L i}, y_{R i}\right]$ containing their WTP-points. Due to rounding, the same intervals can be communicated by different respondents. We consider $n$ as a fixed non-random number.

From our assumptions it follows that we can consider $\mathbf{y}_{1}^{n}=\left\{\mathbf{y}_{1}, \ldots, \mathbf{y}_{n}\right\}$ as a realization of a multinomial random process $\left\{\mathbf{Y}_{i}\right\}_{i \geq 1}$ with the discrete time parameter $i=1,2, \ldots$ The r.v.s $\left\{\mathbf{Y}_{i}\right\}_{i \geq 1}$ are i.i.d. and the set of their values is a set containing all possible self-selected intervals $\mathcal{U}_{\text {all }}=\left\{\mathbf{u}_{\alpha}\right.$ : $\left.P\left[\mathbf{Y}_{i}=\mathbf{u}_{\alpha}\right]>0, \alpha \in A\right\}$. The set $\mathcal{U}_{\text {all }}$ can contain many different intervals $u_{\alpha}$ but due to rounding their number is finite.

There is a discrete probability distribution $p_{\alpha}=P\left[\mathbf{Y}_{i}=\mathbf{u}_{\alpha}\right], \alpha \in A$. The set $\mathcal{U}_{\text {all }}$ and the probability distribution $\left\{p_{\alpha}, \alpha \in A\right\}$ are not known. In our context $\alpha$ is an integer index identifying $\mathbf{u}_{\alpha}$, i.e. $\mathbf{u}_{\alpha^{\prime}} \neq \mathbf{u}_{\alpha^{\prime \prime}}$ if $\alpha^{\prime} \neq \alpha^{\prime \prime}$.

All $m(n) \leq n$ different intervals in $\mathbf{y}_{1}^{n}$ can be ordered by their endpoints. We write $\mathbf{y}_{i_{1}}<\mathbf{y}_{i_{2}}$ if either $y_{L i_{1}}<y_{L i_{2}}$, or $y_{L i_{1}}=y_{L i_{2}}$ but $y_{R i_{1}}<y_{R i_{2}}$. Then $\mathbf{y}_{i_{1}^{\prime}}<\mathbf{y}_{i_{2}^{\prime}}<\ldots<\mathbf{y}_{i_{m(n)}^{\prime}}$ and we let $\mathbf{u}_{h n}=\mathbf{y}_{i_{h}^{\prime}}$ for all $\mathbf{y}_{i}$ identical with $\mathbf{y}_{i_{h}^{\prime}}$.

The collected data can be written as a list:

$$
\mathbf{d} \mathbf{1}_{n}=\left\{\ldots,\left\{h,\left\{u_{L h, n}, u_{R h, n}\right\}, t_{h, n}\right\}, \ldots\right\}
$$

where ordering indexes $h=1, \ldots, m(n)$ of different intervals $\mathbf{u}_{h, n}=\left(u_{L h, n}, u_{R h, n}\right]$ depend on the collected data $\mathbf{y}_{1}^{n}$.

Let $\mathcal{U}_{m(n), n}=\left\{\mathbf{u}_{1, n}, \ldots, \mathbf{u}_{m(n), n}\right\} \subseteq \mathcal{U}_{\text {all }}$ be the set of elicited intervals and 
$\mathbf{t}(n)=\left\{t_{1, n}, \ldots, t_{m(n), n}\right\}$ be the numbers $t_{h, n}=\sum_{i=1}^{n} I\left[\mathbf{y}_{i}=\mathbf{u}_{h, n}\right]$ of times that $\mathbf{u}_{h, n}$ was found in the data $\mathbf{y}_{1}^{n}$.

We are interested in estimating the fraction of respondents (in $\mathfrak{P}$ ) that will state WTP-intervals which are already in the set $\mathcal{U}_{m(n), n}$. Let $p_{c}(n)$ be probability of the event that the last WTP-interval, $\mathbf{y}_{n}=\mathbf{u}_{h_{n}, n}$, in $\mathbf{y}_{1}^{n}$, has been stated by at least one of the previous respondents, $i<n$. Then $t_{h_{n}, n} \geq 2$. Let $H_{n}$ be the r.v. to state a WTP-interval $\mathbf{u}_{H_{n}, n}$ by the $n$-th respondent $\left\{H_{n}=h\right\} \subseteq\left\{X_{i} \in u_{h n}\right\}$. The expectation $E\left[I\left[t_{H_{n}, n} \geq 2\right]\right]=$ $p_{c}(n)$. Therefore $I\left[t_{h_{n}, n} \geq 2\right]$ can be considered as an unbiased estimate of $p_{c}(n)$, and $\mathbf{t}(n)$ is a sufficient statistic with components $t_{h, n}, h=1, \ldots, m(n)$.

Due to uniformly random sampling (simple random sampling without replacement) of respondents from $\mathfrak{P}, \mathbf{t}(n)$ is invariant with respect to any permutation of intervals in $\mathbf{y}_{i}^{n}$. Hence, after averaging $I\left[t_{H_{n}, n} \geq 2\right]$ given $\mathbf{t}(n)$, we obtain the enhanced unbiased estimate of $p_{c}(n)$,

$$
\hat{p}_{c}(n)=r(n) / n,
$$

where $r(n)=\sum_{h=1}^{m(n)} t_{h, n} I\left[t_{h, n} \geq 2\right]$, and cap ^over $p_{c}(n)$ denotes an estimate.

We reduce our problem to a classical urn problem with $r(i)$ white and $i-r(i)$ black balls. Let $\mathcal{U}_{m(i), i}(1)=\left\{\mathbf{u}_{h}: \mathbf{u}_{h} \in \mathcal{U}_{m(i), i}, t_{h i}=1\right\}, \mathcal{U}_{m(i), i}(2)=$ $\left\{\mathbf{u}_{h}: \mathbf{u}_{h} \in \mathcal{U}_{m(i), i}, t_{h i} \geq 2\right\}, \mathcal{U}_{m(i), i}=\mathcal{U}_{m(i), i}(1) \cup \mathcal{U}_{m(i), i}(2)$. If $\mathbf{y}_{i+1} \in$ $\mathcal{U}_{m(i), i}(2)$ then $\Delta_{c}(i)=\hat{p}_{c}(i+1)-\hat{p}_{c}(i)=(i-r(i)) /(i(i+1))$. If $\mathbf{y}_{i+1} \in$ $\mathcal{U}_{m(i), i}(1)$ then $\Delta_{c}(i)=(2 i-r(i)) /(i(i+1))$. If $\mathbf{y}_{i+1} \notin \mathcal{U}_{m(i), i}$ then $\Delta_{c}(i)=$ $-r(i) /(i(i+1))$.

By calculating $\hat{p}_{c}(i)$ for each $i \leq n$ in (3.1) we can observe the evolution of $\hat{p}_{c}(i)$, see Fig. 2 in Section 5 . The probability $p_{c}(n)$ is increasing in $n$. Hence, we can use $\hat{p}_{c}(n)$ as a lower bound estimate for $p_{c}\left(n^{\prime}\right)$, for any $n^{\prime}>n$. Then we may interpret $\hat{p}_{c}(n) 100 \%$ as a lower bound estimate of the percentage of all individuals in $\mathfrak{P}$ who would claim an interval $\mathbf{u}_{h} \in \mathcal{U}_{m(n), n}$. We call $p_{c}(n)$ a coverage probability. 
The decision to stop collecting data on the first step can thus depend

1 on the value of $\hat{p}_{c}(n)$. If this value is not sufficiently close to 1 , and if it is possible to extend data collection, we have information about the value of so doing. Note that if the collection of data has stopped with $n_{1} \geq n$ and $\hat{p}_{c}\left(n_{1}\right)=r\left(n_{1}\right) / n_{1}$ then inferences about the WTP-distribution will only correspond to the subset of individuals who have WTP-intervals in $\mathcal{U}_{m\left(n_{1}\right), n_{1}}$.

Henceforth, the number $n_{1}$ of randomly sampled respondents is fixed and we suppress indexes, e.g. we will write $\mathcal{U}_{m}=\left\{\mathbf{u}_{1}, \ldots, \mathbf{u}_{m}\right\}$ instead of $\mathcal{U}_{m\left(n_{1}\right), n_{1}}=\left\{\mathbf{u}_{1, n_{1}}, \ldots, \mathbf{u}_{m\left(n_{1}\right), n_{1}}\right\}$, and $t_{h}$ instead of $t_{h, n_{1}}$. We write $\mathcal{U}_{m}, \mathbf{u}_{h}, \mathbf{v}_{j}, \mathcal{C}_{h}$ and $\mathcal{D}_{j}$ instead of $\mathcal{U}_{m_{n}, n_{1}}, \mathbf{u}_{h n_{1}}, \mathbf{v}_{j n_{1}}, \mathcal{C}_{h n_{1}}$ and $\mathcal{D}_{j n_{1}}$.

Let $v_{0}<v_{1}<\ldots<v_{k-1}<v_{k}$ be ordered values of the end points of all intervals $\mathbf{u}_{h} \in \mathcal{U}_{m}$. We let $v_{L j}=v_{j-1}$ and $v_{R j}=v_{j}$. Then the intervals in the following collection $\mathcal{V}_{k}=\left\{\mathbf{v}_{1}, \ldots, \mathbf{v}_{j}, \ldots, \mathbf{v}_{k}\right\}, \mathbf{v}_{j}=\left(v_{L j}, v_{R j}\right] j=1, . ., k$, are called division intervals. Each interval $\mathbf{u}_{h} \in \mathcal{U}_{m}$ is the union of disjoint division intervals

$$
\mathbf{u}_{h}=\bigcup_{j \in \mathfrak{C}_{h}} \mathbf{v}_{j}, \quad \mathcal{C}_{h}=\left\{j: \mathbf{v}_{j} \subseteq \mathbf{u}_{h}\right\} .
$$

Below we will also use the following sets of indices $h$,

$$
\mathcal{D}_{j}=\left\{h: \mathbf{v}_{j} \subseteq \mathbf{u}_{h}\right\}, \quad j=1, \ldots, k .
$$

Respondents' WTP-points $\left\{x_{i}\right\}$ are values of i.i.d. r.v.s $\left\{X_{i}\right\}$. We need to estimate the d.f. $F[x]=P\left[X_{i} \leq x\right]$, or equivalently, the survival function (s.f.) $S[x]=1-F[x]$ of the true WTP-distribution. Let $H_{i}, i=1, \ldots, n_{1}$, be i.i.d. r.v.s, $H_{i}=h$ given that, on the first step, the $i$-th respondent has stated the interval $\mathbf{u}_{h}$ containing his/her WTP-point $x_{i},\left\{H_{i}=h\right\} \subseteq\left\{X_{i} \in \mathbf{u}_{h}\right\}$. We let $w_{h}=P\left[\left\{H_{i}=h\right\} \cap\left\{X_{i} \in \mathbf{u}_{h}\right\}\right], w_{h} \leq p_{h}=P\left[X_{i} \in \mathbf{u}_{h}\right], h=1, \ldots, m$.

The events $\left\{H_{i}=h\right\} \cap\left\{X_{i} \in \mathbf{u}_{h}\right\}$ are observable, while events $\left\{X_{i} \in\right.$ $\left.\mathbf{u}_{h^{\prime}}\right\}, h^{\prime} \neq h$, are not. The probability of the number of times $t_{1}, \ldots, t_{m}$ that 
$\mathbf{u}_{1}, \ldots, \mathbf{u}_{m}$ appears is $\prod_{h=1}^{m} w_{h}^{t_{h}}$, i.e. we have a multinomial distribution. The corresponding normed log likelihood (llik) is

$$
\operatorname{llik}\left[w_{1}, \ldots, w_{m} \mid t_{1}, \ldots, t_{m}\right]=\sum_{h=1}^{m} \frac{t_{h}}{n_{1}} \log \left[w_{h}\right], \quad \sum_{h=1}^{m} t_{h}=n_{1} .
$$

The maximum of llik over $w_{h} \geq 0, \sum_{h=1}^{m} w_{h}=1$, is attained at $\check{w}_{h}=$ $t_{h} / n_{1}, h=1, \ldots, m$. Note that $-\sum_{h=1}^{m} \check{w}_{h} \log \left[\hat{w}_{h}\right]$ is the empirical Entropy of the multinomial distribution with probabilities $\left\{\hat{w}_{1}, \ldots, \hat{w}_{m}\right\}$.

Let us now introduce the second step of data collection. We prolong random sampling of new (not yet sampled) individuals from the population $\mathfrak{P}$. In the second step each individual is to announce an interval containing his/her WTP-point. If the interval does not belong to $\mathfrak{U}_{m}$ then we do not include it in the collected data. If the interval $\mathbf{u}_{h}$ belongs to $\mathcal{U}_{m}$ then this respondent is asked to select an interval $\mathbf{v}_{j} \in \mathcal{V}_{k}, \mathbf{v}_{j} \subseteq \mathbf{u}_{h}$, from the division $V_{k}$ containing his/her true WTP-point. The respondents may well abstain from answering this second question and we will cater for these events in the collected data.

The collected data will be the list of triples $\mathbf{d} \mathbf{2}_{n \cdot 2}=\left\{\mathbf{z}_{1}, \ldots, \mathbf{z}_{n \cdot 2}\right\}, \mathbf{z}_{i}=$ $\left\{i, \mathbf{u}_{h_{i}}, N A\right\}$ or $\left\{i, \mathbf{u}_{h_{i}}, \mathbf{v}_{j_{i}}\right\}, N A$ is "no answer" to the additional question; we call these triples singles and pairs. We suppose that $n_{2}$ is sufficiently large and that any $\mathbf{v}_{j} \in \mathcal{V}_{k}$ was reported sufficiently many times. The size $n_{.2}$ of the data collected in the second step should be significantly larger than $n_{1}$.

\section{STATISTICAL MODEL}

Henceforth we consider r.v.s $X_{i}$ and probabilities of events for individuals who being asked would have intervals included in $\mathcal{U}_{m}$.

Let us consider the conditional probabilities

$$
w_{h j}=P\left[H_{i}=h \mid X_{i} \in \mathbf{v}_{j}\right] .
$$


We call a pair of $h, j$ compatible if $\mathbf{v}_{j} \subseteq \mathbf{u}_{h}$. It is not possible to identify the true WTP-distribution if we only have the data $\mathbf{d} \mathbf{1}_{n_{1}}$ obtained on the first step. For identification of the true WTP-distribution on the division intervals $\mathcal{V}_{k}$ it is necessary to consistently estimate the conditional probabilities $\mathbb{W}=\left(w_{h j}\right)$ by using data collected on the second step. We obtain this identification asymptotically as the size of data collected in the second step is growing without bounds. The values of probabilities $w_{h j}$ for a given $j=1, \ldots, k$ reflect behavior of individual's selecting $\mathbf{u}_{h} \supseteq \mathbf{v}_{j}$, if $X_{i}=x_{i} \in \mathbf{v}_{j}$. By the formula of total probability we can write

$$
w_{t r h}=\sum_{j \in \mathrm{e}_{h}} w_{h j} q_{t r j},
$$

where $q_{t r j}=P\left[X_{i} \in \mathbf{v}_{j}\right]$, is the true probability of the event $\left\{\mathbf{X}_{i} \in \mathbf{v}_{j}\right\}$.

We have no information about the process of selecting intervals but our approach avoids the necessity of knowing the true behavioral model. The collected data with pairs $\left\{i, \mathbf{u}_{h_{i}}, \mathbf{v}_{j_{i}}\right\}$ provides a simple way to estimate $q_{t r j}=P\left[X_{i} \in \mathbf{v}_{j}\right], j=1, \ldots, k$. Let $c_{p j}=\sum_{i=1}^{n \cdot 2} I\left[\mathbf{z}_{i}=\left\{i, \mathbf{u}_{h_{i}}, \mathbf{v}_{j}\right\}\right], \quad c_{p h j}=$ $\sum_{i=1}^{n .2} I\left[\mathbf{z}_{i}=\left\{i, \mathbf{u}_{h}, \mathbf{v}_{j}\right\}\right], n_{s 2}=\sum_{h=1}^{m} t_{s h}, n_{p 2}=\sum_{j=1}^{k} c_{p j}, n_{\cdot 2}=n_{s 2}+n_{p 2}$. The sub-indeces $s$ and $p$ correspond to singles and pairs, $t_{s h}=\sum_{i=1}^{n .2} I\left[\mathbf{z}_{i}=\right.$ $\left.\left\{i, \mathbf{u}_{h}, N A\right\}\right]$.

We consider $n_{p 2} / n_{.2}$ as a frequency in the Binomial process with singles and pairs and $n_{p 2} / n_{\cdot 2} \rightarrow \alpha_{p} \in(0,1)$ almost surely (a.s.) as $n_{\cdot 2} \rightarrow \infty$. We can also consider the conditional Binomial process with pairs containing $\mathbf{v}_{j}$ together with any $\mathbf{u}_{h} \supseteq \mathbf{v}_{j}, \mathbf{u}_{h} \in \mathcal{U}_{m}$. To reduce notational complexity for division intervals with $q_{t r j}=0$, we consider the case when all $q_{t r j}>0, j=$ $1, \ldots, k$. The cases with some of $q_{t r j}=0$ may be considered similarly.

The strongly consistent estimates of $q_{t r j}$ in (4.1) are

$$
\check{q}_{p j}=\frac{c_{p j}}{n_{p 2}} \rightarrow q_{t r j}, \quad \text { a.s. } \quad j=1, \ldots, k, \text { as } n_{\cdot 2} \rightarrow \infty .
$$

We can also use the pairs $\left\{\mathbf{u}_{h_{i}}, \mathbf{v}_{j_{i}}\right\}$ to obtain the following strongly consis- 
tent estimates of $w_{h j}$

$$
\hat{W}_{m k}=\left\{\hat{w}_{h j}=\frac{c_{p h j}}{c_{p j}}, \quad h \in \mathcal{D}_{j}, \quad j=1, \ldots, k\right\} .
$$

2

In (4.3) $c_{p h j}$ is the number of saved pairs $\left\{i, \mathbf{u}_{h}, \mathbf{v}_{j}\right\}, h \in \mathcal{D}_{j}$, in the data $\mathbf{d} \boldsymbol{2}_{n \cdot 2} . \mathcal{D}_{j}$ is defined in (3.3). Note that $\sum_{h=1}^{m} \hat{w}_{h j}=1$ for each $j=1, \ldots, k$.

Our approach is nonparametric in the sense that we do not assume that $q_{j}, j=1, \ldots, k$ are known functions of some parameters. In addition, we do not impose any restrictions on the behavior of respondents, in terms of the relative position of their true WTP-points inside the (potentially rounded) intervals.

Note that $\mathbf{q}_{k}=\left\{q_{1}, \ldots, q_{k}\right\}$ is a point in the polyhedron $\mathcal{S}_{k-1}$

$$
\mathcal{S}_{k-1}=\left\{\mathbf{q}_{k}: 0 \leq q_{j} \leq 1, \sum_{j=1}^{k} q_{j}=1\right\}
$$

The log likelihood related to the saved data from the $i$ th respondent is

$$
\begin{aligned}
l_{i}\left[\mathbf{q}_{k} \mid \hat{\mathbb{W}}_{m k}, \mathbf{d} \mathbf{2}_{n \cdot 2}\right] & =I\left[z_{i}=\left\{i, \mathbf{u}_{h_{i}}, N A\right\}\right] \log \left[\sum_{j \in \mathfrak{C}_{h_{i}}} \hat{w}_{h_{i} j} q_{j}\right] \\
& +I\left[z_{i}=\left\{i, \mathbf{u}_{h_{i}}, \mathbf{v}_{j_{i}}\right\}\right] \log \left[\hat{w}_{h_{i} j_{i}} q_{j_{i}}\right],
\end{aligned}
$$

where $\hat{w}_{h j}$ and $\mathcal{C}_{h_{i}}$ are defined in (4.3) and (3.2), respectively. In short we write $l_{i}\left[\mathbf{q}_{k}\right]$ instead of $\mathrm{ll}_{i}\left[\mathbf{q}_{k} \mid \hat{\mathbb{W}}_{m k}, \mathbf{d} \boldsymbol{2}_{n \cdot 2}\right]$.

Now we can write the following, normed by $n_{\cdot 2}=n_{s 2}+n_{p 2}$, log likelihood function corresponding to the data $\mathbf{d} \mathbf{2}_{n_{.2}}$ containing both singles and pairs

$$
\operatorname{lik}\left[\mathbf{q}_{k} \mid \hat{\mathbb{W}}_{m k}, \mathbf{d} \boldsymbol{2}_{n \cdot 2}\right]=\frac{1}{n_{\cdot 2}} \sum_{i=1}^{n_{\cdot 2}} \operatorname{ll}_{i}\left[\mathbf{q}_{k}\right]
$$

From (4.5) and (4.6) we obtain the following estimate of the normed by $n_{\cdot 2} \log$ likelihood function of $\mathbf{q}_{k}=\left\{q_{1}, \ldots, q_{k}\right\}$ corresponding to the all data, 5 6 
with pairs and singles, collected on the second step

1

$$
\begin{aligned}
\operatorname{llik}\left[\mathbf{q}_{k} \quad \mid \quad \hat{W}_{m k}, \mathbf{d} \boldsymbol{2}_{n_{\cdot 2}}\right]=\frac{n_{s 2}}{n_{\cdot 2}} \sum_{h=1}^{m} \frac{t_{s h}}{n_{s 2}} \log \left[\sum_{j \in \mathcal{C}_{h}} \hat{w}_{h j} q_{j}\right] \\
+\frac{n_{p 2}}{n_{\cdot 2}} \sum_{j=1}^{k} \frac{c_{p j}}{n_{p 2}} \log \left[q_{j}\right]+\frac{1}{n_{\cdot 2}} \sum_{i=1}^{n_{2}} \log \left[\hat{w}_{h_{i} j_{i}}\right] I\left[\mathbf{z}_{i}=\left\{i, \mathbf{u}_{h_{i}}, \mathbf{v}_{j_{i}}\right\}\right] .
\end{aligned}
$$

Let $\partial \mathcal{S}_{k-1}$ be all boundary points of $\mathcal{S}_{k-1}$. The log likelihood function (4.7) is concave on $\mathcal{S}_{k-1} \backslash \partial \mathcal{S}_{k-1}$, see Theorem 7.1 in Appendix.

We have obtained (see Appendix) the following recursion, $r=1,2, \ldots$,

$$
q_{j}^{(r+1)}=\frac{n_{p 2}}{n_{s 2}+n_{p 2}} q_{j}^{(1)}+\frac{n_{s 2}}{n_{s 2}+n_{p 2}} \sum_{h \in \mathcal{D}_{j}} \frac{\hat{w}_{h j} q_{j}^{(r)}}{\sum_{j^{\prime} \in \mathcal{C}_{h}} \hat{w}_{h j^{\prime}} q_{j^{\prime}}^{(r)}} \hat{w}_{s h},
$$

$$
q_{j}^{(1)}=\check{q}_{p j}, \quad \hat{w}_{s h}=\frac{1}{n_{s 2}} \sum_{i=1}^{n \cdot 2} I\left[z_{i}=\left\{i, \mathbf{u}_{h}, N A\right\}\right] .
$$

$q_{j}^{(1)}=q_{j}^{(1)}\left(n_{p 2}\right), q_{j}^{(r)}=q_{j}^{(r)}\left(n_{p 2}, n_{s 2}\right), \hat{w}_{s h}=\hat{w}_{s h}\left(n_{s 2}\right), j=1, \ldots, k, h=1, \ldots, m$, $r=2,3, \ldots$. If $n_{p 2}$ and $n_{s 2}$ are sufficiently large then

$$
\hat{w}_{s h}>0, \quad \sum_{j^{\prime} \in \mathfrak{e}_{h}} \hat{w}_{h j^{\prime}} q_{j}^{(1)}>0 \quad \text { and } \quad q_{j}^{(1)}=\check{q}_{p j}>0
$$

for each $h=1, \ldots, m, j=1, \ldots, k$. If (4.9) holds then the recursion (4.8) can be applied for obtaining the ML-estimators of probabilities $\mathbf{q}_{t r}$ maximizing llik (4.7).

THEOREM 4.1 Suppose that Assumptions 1-3 are valid and the sizes $n_{p 2}$ and $n_{s 2}$ of collected pairs and singles are growing unboundedly as $n_{\cdot 2} \rightarrow$ $\infty$. Then for any sufficiently large $n_{p 2}$ and $n_{s 2}$ the $M L$-estimator $\check{\mathbf{q}}_{k}=$ $\left\{\check{q}_{1}, \ldots, \check{q}_{k}\right\}$ (based on the data $\mathbf{d} \mathbf{2}_{n \cdot 2}$ with singles and pairs) of the log likelihood (4.7) exists, is strongly consistent, and can be found as the stationary point of recursion (4.8).

Proof. From (4.2) and (4.8) it follows that $q_{j}^{(1)} \rightarrow q_{t r j}$ a.s. $n_{.2} \rightarrow \infty, j=$ $1, \ldots, k, \mathbf{q}_{t r k} \in \mathcal{K}_{\alpha_{p}}=\left\{\mathbf{q}_{k}: q_{j} \geq q_{t r j} \alpha_{p} / 2, \sum_{j=1}^{k} q_{j}=1\right\} \subset \mathcal{S}_{k-1} \backslash \partial \mathcal{S}_{k-1}$. 
For all sufficiently large $n_{\cdot 2}$ all $\mathbf{q}_{k}^{(r)} \in \mathcal{K}_{\alpha_{p}}, r \geq 1$. Due to concavity of (4.7) the sequence $\left\{\mathbf{q}_{k}^{(r)}\right\}_{r \geq 1}$ has only one limit stationary point of the recursion (4.8) in the compact set $\mathcal{K}_{\alpha_{p}}$ where (4.7) has its maximum. We summarize these results. From Theorem 7.1, Corollaries 7.2 - 7.3 follows existence of the ML-estimates $\check{\mathbf{q}}_{k}=\check{\mathbf{q}}_{k M}$ which converge a.s. to $\mathbf{q}_{t r k}$. Corollary 7.2 justifies usage of the recursion (4.8) to approximate the ML-estimates $\check{\mathbf{q}}_{k}$.

Hence, we can find consistent ML-estimates $\check{q}_{j}, j=1, \ldots, k$, of the projected WTP-distribution on the division intervals $\mathbf{v}_{j} \in \mathcal{V}_{k}$, i.e. $\check{q}_{j} \rightarrow q_{t r j}$ a.s. $j=1, \ldots, k$, if $n_{p 2}$ and $n_{s 2}$ are growing. Note that this inference can be only applied to the respondents in $\mathfrak{P}$ who choose $\mathbf{u}_{h} \in \mathcal{U}_{m}$.

Besides consistency we need to know the accuracy of the obtained MLestimate $\check{\mathbf{q}}_{k}$, or $\check{\mathbf{q}}_{k-1}=\left\{\check{q}_{1}, \ldots, \check{q}_{k-1}\right\}, \check{q}_{k}=1-\sum_{j=1}^{k-1} \check{q}_{j}$. In our case we are interested in consistently estimating the distribution of rescaled deviations $\sqrt{n \cdot 2}\left(\check{\mathbf{q}}_{k-1}-\mathbf{q}_{t r, k-1}\right)$,

$$
Q_{q_{t r, k-1}}\left[x_{1}, \ldots, x_{k-1} \mid \hat{\mathbb{W}}_{m k}, \mathbf{d} \boldsymbol{2}_{n \cdot 2}\right]=P\left[\bigcap_{j=1}^{k-1}\left\{\sqrt{n_{\cdot 2}}\left(\check{q}_{j}-q_{t r j}\right) \leq x_{j}\right\}\right] .
$$

The log likelihoods $\mathrm{ll}_{i}\left[\mathbf{q}_{k}\right]$ in (4.5) are values of r.v.s

$$
\begin{aligned}
\mathrm{LL}_{i}\left[\mathbf{q}_{k}\right] & =I\left[Z_{i}=\left\{i, \mathbf{u}_{H_{i}}, N A\right\}\right] \log \left[\sum_{j \in \mathfrak{C}_{H_{i}}} \hat{w}_{H_{i} j} q_{j}\right] \\
& +I\left[Z_{i}=\left\{i, \mathbf{u}_{H_{i}}, \mathbf{v}_{J_{i}}\right\}\right] \log \left[\hat{w}_{H_{i} J_{i}} q_{J_{i}}\right], \quad i=1, \ldots, n_{\cdot 2},
\end{aligned}
$$

where $H_{i}$ and $J_{i}$ are random indexes of $\mathbf{u}_{H_{i}}$ and $\mathbf{v}_{J_{i}}$. The asymptotics of deviations ML-estimates in parametric statistical models with independent observations are rather completely investigated if regularity properties (RP), of the observations' $\log$ likelihoods $\mathrm{LL}_{i}\left[\mathbf{q}_{k}\right]$, hold (Lehmann and Casella (1998)). In our case these RP can be described as follows.

RP1: all first and second order partial derivatives

$$
\frac{\partial \mathrm{LL}_{i}\left[\tilde{\mathbf{q}}_{k}\right]}{\partial q_{j_{1}}}, \frac{\partial^{2} \mathrm{LL}_{i}\left[\left[\tilde{\mathbf{q}}_{k}\right]\right.}{\partial q_{j_{1}} \partial q_{j_{2}}}, \quad 1 \leq j_{1}, j_{2} \leq k-1, \quad \tilde{\mathbf{q}}_{k}=\left\{q_{1}, \ldots, q_{k-1}, 1-\sum_{j=1}^{k-1} q_{j}\right\}
$$


exist and are continuous functions on the set of all possible values $\mathbf{q}_{k}$.

1

RP2: these derivatives possess finite absolute moments of an order $\gamma>2$.

RP3: the second order partial derivatives are uniformly continuous on any containing $\mathbf{q}_{t r k}$ compact set of possible values $\mathbf{q}_{k}$,

$\mathbf{R P} 4$ : for all $1 \leq j_{1}, j_{2} \leq k-1$

$$
E_{\mathbf{q}_{t r k}}\left[\frac{\partial \mathrm{LL}_{i}\left[\tilde{\mathbf{q}}_{k}\right]}{\partial q_{j_{1}}}\right]=0,
$$$$
E_{\mathbf{q}_{t r k}}\left[\frac{\partial^{2} \mathrm{LL}_{i}\left[\tilde{\mathbf{q}}_{k}\right]}{\partial q_{j_{1}} \partial q_{j_{2}}}\right]=-E_{\mathbf{q}_{t r k}}\left[\frac{\partial \mathrm{LL}_{i}\left[\tilde{\mathbf{q}}_{k}\right]}{\partial q_{j_{1}}} \frac{\partial \mathrm{LL}_{i}\left[\tilde{\mathbf{q}}_{k}\right]}{\partial q_{j_{2}}}\right] .
$$

In order to formulate RP5 we introduce the Fisher information (FI-) matrices

$$
\mathbb{F}_{n \cdot 2}\left[\tilde{\mathbf{q}}_{k}\right]=\frac{1}{n \cdot 2} \sum_{j=1}^{n \cdot 2}\left(E_{\mathbf{q}_{t r, k-1}}\left[\frac{\partial^{2} \mathrm{LL}_{i}\left[\mathbf{q}_{k}\right]}{\partial q_{j_{1}} \partial q_{j_{2}}}\right]\right)_{1 \leq j_{1} \leq j_{2} \leq k-1} .
$$

RP5: for each possible $\mathbf{q}_{k}$ the FI-matrix has full rank $k-1$.

In Appendix we show that RP1 - RP5 are valid for the statistical model with $\log$ likelihood (4.7). These properties imply the standard regularity properties.

The distributions, of rescaled deviations of ML-estimators from true values of parameters, thus converge to normal distributions with the inverse FI-matrix (Lehmann and Casella (1998)). In our case, with large number $(k-1)$ of parameters to be estimated, it is more convenient to use an universal method for assessing accuracies of estimated parameters based on resamplings (Davison and Hinkley (1995); Belyaev and Nilsson (1997); Nilsson (1998); Belyaev (2007)). We proceed by outlining a procedure for resampling in the considered statistical model of the data $\mathrm{d} \boldsymbol{2}_{n \cdot 2}$.

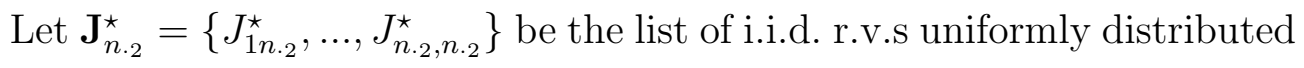
over the range of natural numbers $\{1,2, \ldots, n .2\}$, i.e. $P^{\star}\left[J_{i^{\prime} n \cdot 2}^{\star}=i\right]=\frac{1}{n \cdot 2}, 1 \leq$ $i^{\prime} \leq n .2$. We use "* " to denote r.v.s, probabilities of events, mathematical 
expectations, etc., related to the r.v.s $J_{i n \cdot 2}^{\star}, 1 \leq i \leq n_{.2}$. The values of these r.v.s can be simulated via a random numbers generator.

It is of interest to simulate resampled, with replacement, copies of data $\mathbf{d} 2_{n_{.2}}$. Let $\left\{j_{1 n_{\cdot 2}}^{\star c}, \ldots, j_{n \cdot 2}^{\star c}, n_{\cdot 2}\right\}$, be the $c$-th resampled copy of the list $\left\{1, \ldots, n_{\cdot 2}\right\}$, obtained by simulation i.i.d. values of (pseudo) r.v.s $J_{i n .2}^{\star c}$. Then the $c$-th copy of $\mathbf{d} \mathbf{2}_{n \cdot 2}$ is the list $\mathbf{d} \mathbf{2}_{n \cdot 2}^{\star c}=\left\{\mathbf{z}_{1}^{\star c}, \ldots, \mathbf{z}_{n \cdot 2}^{\star c}\right\}$, where triples $\mathbf{z}_{i}^{\star c}=\mathbf{z}_{i^{\star c}}, \tilde{i}^{\star c}=$ $j_{i n \cdot 2}^{\star c}, i=1, \ldots, n_{\cdot 2}$. We can consider the list $\mathbf{d} \mathbf{2}_{n \cdot 2}^{\star c}$ as a realisation of a multinomial process with the resampled log likelihood

$$
l l i k^{\star c}\left[\mathbf{q}_{k} \mid \hat{\mathbb{W}}_{m k}, \mathbf{d} 2_{n \cdot 2}^{\star c}\right]=\sum_{i=1}^{n \cdot 2} \tilde{n}_{i, n \cdot 2}^{\star c} l_{i}\left[\mathbf{q}_{k}\right]
$$

where $l_{i}\left[\mathbf{q}_{k}\right]$ are defined in (4.5), and $\tilde{n}_{i, \tilde{n} \cdot 2}^{\star c}=\sum_{i=1}^{n \cdot 2} I\left[\tilde{i}^{\star c}=i\right]$.

All methods exposed in Appendix can be applied to the analysis of the statistical model with data $\mathbf{d} \mathbf{2}_{n \cdot 2}^{\star c}$ and the $\log$ likelihood (4.12). We can do that with the following notations:

$$
\tilde{n}_{s 2}^{\star c}=\sum_{i=1}^{n \cdot 2} I\left[\mathbf{z}_{i}^{\star c}=\left\{\tilde{i}^{\star c}, \mathbf{u}_{\tilde{i}^{\star} \star c}, N A\right\}\right],
$$

$$
\tilde{n}_{p 2}^{\star c}=\sum_{i=1}^{n \cdot 2} I\left[\mathbf{z}_{i}^{\star c}=\left\{\tilde{i}^{\star c}, \mathbf{u}_{h_{\tilde{i}^{\star c}}}, \mathbf{v}_{\tilde{i}_{\tilde{i}^{\star}}}\right\}\right],
$$$$
\tilde{n}_{s h}^{\star c}=\sum_{i=1}^{n \cdot 2} I\left[\mathbf{z}_{i}^{\star c}=\left\{\tilde{i}^{\star c}, \mathbf{u}_{h}, N A\right\}\right],
$$

$$
\tilde{n}_{p h j}^{\star c}=\sum_{i=1}^{n \cdot 2} I\left[\mathbf{z}_{i}^{\star c}=\left\{\tilde{i}^{\star c}, \mathbf{u}_{h}, \mathbf{v}_{j}\right\}\right]
$$
(a) 
We can rewrite the log likelihood (4.12) as follows

$$
\begin{aligned}
& l l i k^{\star c}\left[\mathbf{q}_{k} \mid \hat{\mathbb{W}}_{m k}, \mathbf{d} \mathbf{2}_{n_{2}}^{\star c}\right] \\
= & \frac{\tilde{n}_{s 2}^{\star c}}{n_{\cdot 2}} \sum_{h=1}^{m} \frac{t_{s h}^{\star c}}{n_{s 2}^{\star c}} \log \left[\sum_{j \in \mathcal{C}_{h}} \hat{w}_{h j} q_{j}\right]+\frac{\tilde{n}_{p 2}^{\star c}}{n_{\cdot 2}} \sum_{j=1}^{k} \frac{t_{p j}^{\star c}}{n_{p 2}^{\star c}} \log \left[q_{j}\right] \\
+ & \frac{\tilde{n}_{p 2}^{\star c}}{n_{\cdot 2}} \sum_{j=1}^{k} \sum_{j \in \mathcal{D}_{j}} \frac{t_{p h j}^{\star c}}{\tilde{n}_{p 2}^{\star c}} \log \left[w_{h j}\right],
\end{aligned}
$$

The recursion corresponding the log likelihood (4.13) is

$$
q_{j}^{\star c(r+1)}=\frac{\tilde{n}_{p 2}^{\star c}}{n \cdot 2} q_{j}^{\star c(1)}+\frac{\tilde{n}_{s 2}^{\star c}}{n \cdot 2} \sum_{h \in \mathcal{D}_{j}} \frac{t_{s h}^{\star c}}{\tilde{n}_{s 2}^{\star c}} \frac{\hat{w}_{h j} q_{j}^{\star c(r)}}{\sum_{j^{\prime} \in \mathcal{C}_{h}} \hat{w}_{h j^{\prime}} q_{j^{\prime}}^{\star c(r)}}, \quad j=1, \ldots, k,
$$
(4.14) as $n_{\cdot 2} \rightarrow \infty$.

Theorem 7.1 and 7.2, Corollaries $7.1-7.3$ and regularity properties RP1

- RP5 are valid for resampled data $\mathbf{d} \mathbf{2}_{n \cdot 2}^{\star c}$ with the log likelihood (4.13). Hence, the following Corollary is valid.

Corollary 4.1 Statistical model with resampled data $\mathbf{d} \mathbf{2}_{n \cdot 2}^{\star c}$ and the $\log$ likelihood function (4.12) possesses regularity properties RP1 - RP5 and has consistent ML-estimator $\check{\mathbf{q}}_{k}^{\star c}-\mathbf{q}_{t r k} \rightarrow 0$, as $n_{\cdot 2} \rightarrow \infty$.

In order to estimate the distribution of deviations $Q_{\mathbf{q}_{t r, k-1}}\left[x_{1}, \ldots, x_{k-1} \mid\right.$ $\left.\mathbb{W}_{m k}, \mathbf{d} \boldsymbol{2}_{n .2}\right]$ defined in (4.10) we need to simulate sufficiently large number $R$ of data copies $\mathbf{d} \mathbf{2}_{n \cdot 2}^{\star c}$ and find corresponding ML-estimates $\check{\mathbf{q}}_{k}^{\star c}, c=1, \ldots, R$. Let

$$
Q_{n \cdot 2, R}^{\star}\left[x_{1}, \ldots, x_{k-1}\right]=\frac{1}{R} \sum_{c=1}^{R} \prod_{j=1}^{k-1} I\left[\sqrt{n \cdot 2}\left(\check{q}_{j}^{\star c}-\check{q}_{j}\right) \leq x_{j}\right]
$$

where $\check{q}_{j}$ is the $j$ th component in the vector ML-estimate $\check{\mathbf{q}}_{k M}$ of $\mathbf{q}_{t r k}$.

Theorem 4.2 Suppose that Assumptions 1 - 3 are valid. Then the components of log likelihood (4.6) and (4.12) possess the regularity properties, 
and for any real values $x_{1}, \ldots, x_{k-1}$

1

$$
\sup _{x_{1}, \ldots, x_{k-1}}\left|Q_{n \cdot 2, R}^{\star}\left[x_{1}, \ldots, x_{k-1}\right]-Q_{t r, k-1}\left[x_{1}, \ldots, x_{k-1}\right]\right| \rightarrow 0,
$$

in probability as $n_{\cdot 2} \rightarrow \infty$ and $R \rightarrow \infty$.

Proof. From Theorem 4.1 and Corollary 4.1 we have that in the considered original statistical model with data $\mathbf{d} \boldsymbol{2}_{n \cdot 2}$ and in the statistical models with resampled data $\mathbf{d} \mathbf{2}_{n \cdot 2}^{\star c}$ exist consistent ML-estimates $\check{\mathbf{q}}_{k M}$ and $\check{\mathbf{q}}_{k}^{\star c}$ for all sufficiently large $n_{\cdot 2}$. Properties $\mathbf{P D}, \mathbf{P E}, \mathbf{P R}$ and $\mathbf{P D}^{\star}, \mathbf{P} \mathbf{E}^{\star}, \mathbf{P} \mathbf{R}^{\star}$ are also valid (see Appendix). Then the statement in Theorem 4.2 is a special case of Proposition 2 in Appendix. It follows that the distributions of deviations $\sqrt{n \cdot 2}\left(\check{\mathbf{q}}_{k M}-\mathbf{q}_{t r k}\right)$ and $\sqrt{n \cdot 2}\left(\check{\mathbf{q}}_{k M}^{\star c}-\check{\mathbf{q}}_{k M}\right)$ are approaching in probability as $n_{2} \rightarrow \infty$. These distributions of deviations are asymptotically normal and therefore convergence in the uniform metric stated in (4.16) is valid.

Mean WTP, i.e. $m_{t r 1}=E\left[X_{i}\right]$, is an essential parameter in cost-benefit analysis. Here, however, the rounding of WTP-intervals excludes the possibility to find an unbiased estimate of $m_{t r 1}$. Therefore, we introduce an approximation to $m_{1 t r}$ by the following consistently estimable medium mean of the true WTP-distribution

$$
m m_{1 t r}=v_{L 1}+\sum_{j=1}^{k}\left(q_{t r, j+1}^{k}+\frac{1}{2} q_{t r j}\right)\left(v_{R j}-v_{L j}\right),
$$

where $q_{t r, j+1}^{k}=\sum_{i=j+1}^{k} q_{t r i}, q_{t r, k+1}=0$.

We can also consistently estimate the low $m_{1 t r}^{-}$and upper $m_{1 t r}^{+}$boundaries for $m_{1 t r}$. Let $q_{j}^{k}=q_{j}+\ldots+q_{k}, q_{k}^{k}=q_{k}, q_{k+1}^{k}=0, q_{1}^{k}=1$. Hence, we have

$$
m_{1 t r}^{-}=v_{L 1}+\sum_{j=1}^{k-1} q_{j+1, t r}^{k}\left(v_{R j}-v_{L j}\right), \quad m_{1 t r}^{+}=v_{L 1}+\sum_{j=1}^{k} q_{j, t r}^{k}\left(v_{R j}-v_{L j}\right) .
$$

From (4.17), (4.18) we have

$$
m_{1 t r}^{-} \leq m_{1 t r} \leq m_{1 t r}^{+}, \quad m_{1 t}^{-} \leq m m_{1 t r} \leq m_{1 t r}^{+} .
$$


Note that $m_{1 t r}^{-}, m_{1 t r}^{+}$and $m m_{1 t r}$ are linear forms of $q_{j t r}, j=1, \ldots, k$. We introduced $m m_{1 t r}$ because it better approximates $m_{1 t r}$ than $m_{1 t r}^{-}$and $m_{1 t r}^{+}$. Under some additional assumptions on the true WTP-distribution the estimate $m m_{1 t r}$ can be rather close to $m_{1 t r}$. The characteristic $m m_{1 t r}$ can be consistently estimated and its accuracy can be also consistently estimated as $n_{2} \rightarrow \infty$. The same is also true for the low and upper boundaries $m_{1 t r}^{+}, m_{1 t r}^{-}$. To underline dependency of the considered characteristics of data size $n .2$ we add it into the indices of considered characteristics. We obtain consistent estimators substituting for all $q_{j}$ the corresponding ML-estimates $\check{q}_{j n .2}, j=1, \ldots, k$.

The characteristics (4.17) and (4.18) are linear forms of $\check{q}_{j}=\check{q}_{j n .2}, j=$ $1, \ldots, k$. Hence, by substitution ML-estimates $\check{\mathbf{q}}_{k n \cdot 2}$ instead of $\mathbf{q}_{k}$ in (4.18) we obtain the following strongly consistent ML-estimates of $\check{m}_{1 n \cdot 2}^{+}, \check{m}_{1 n \cdot 2}^{-}$and $m_{m} m_{1 n \cdot 2}$

$$
\check{m}_{1 n \cdot 2}^{-}=v_{L 1}+\sum_{j=1}^{k-1} \check{q}_{j+1, n \cdot 2}^{k}\left(v_{R j}-v_{L j}\right), \quad \check{m}_{1 n \cdot 2}^{+}=v_{R 1}+\sum_{j=1}^{k} \check{q}_{j, n \cdot 2}^{k}\left(v_{R j}-v_{L j}\right) .
$$

We have the following strongly consistent ML-estimate of $m m_{1 t r}$

$$
\operatorname{mm}_{1 n \cdot 2}=v_{L 1}+\sum_{j=1}^{k}\left(\check{q}_{j+1, n \cdot 2}^{k}+\frac{1}{2} \check{q}_{j n \cdot 2}\right)\left(v_{R j}-v_{L j}\right)
$$

$\check{q}_{j+1, n \cdot 2}^{k}=\sum_{i=j+1}^{k} \check{q}_{i n \cdot 2}, j=1, \ldots, k, \check{q}_{k+1, n \cdot 2}=0$. Note that $\check{m m}_{1 n \cdot 2}$ is the area below the broken line, with points $\left\{v_{R j}, \check{q}_{j+1, n \cdot 2}^{k}\right\}, j=1, \ldots, k-1$ and $\left\{v_{R k}, 0\right\}$ on the estimated WTP-survival function, connected by intervals.

By replacing in (4.19) and (4.20) the estimates $\check{q}_{j n .2}$ by $q_{j n .2}^{\star c}, j=1, \ldots, k$, we obtain copies of ML-estimates based on the resampled log likelihoods $(4.12)$

$$
\check{m}_{1 n \cdot 2}^{-\star c}=v_{L 1}+\sum_{j=1}^{k-1} \check{q}_{j+1, n \cdot 2}^{\star c k}\left(v_{R j}-v_{L j}\right)
$$


1

2

3

4

5

$$
\check{m}_{1 n \cdot 2}^{+\star c}=v_{L 1}+\sum_{j=1}^{k} \check{q}_{j, n \cdot 2}^{\star c k}\left(v_{R j}-v_{L j}\right)
$$

$$
m^{\check{m}} m_{1 n \cdot 2}^{\star c}=v_{L 1}+\sum_{j=1}^{k}\left(\check{q}_{j+1, n \cdot 2}^{\star c k}+\frac{1}{2} \check{q}_{j, n \cdot 2}^{\star c}\right)\left(v_{R j}-v_{L j}\right)
$$

All these estimates (4.19) - (4.21) are linear forms w.r.t. $q_{j t r}, \check{q}_{j n .2}$ and $\check{q}_{j n \cdot 2}^{\star c}$. Therefore, from Theorem 4.2 we obtain

COROLlary 4.2 If Assumptions 1 - 3 are valid, then the regularity properties of components in log likelihood (4.6), (4.12) are also valid and

$$
\frac{1}{C} \sum_{c=1}^{C} I\left[\sqrt{n \cdot 2}\left(\check{m}_{1 n \cdot 2}^{-\star c}-\check{m}_{1 n \cdot 2}^{-}\right) \leq x\right]-P\left[\sqrt{n_{\cdot 2}}\left(\check{m}_{1 n \cdot 2}^{-}-m_{1 t r}^{-}\right) \leq x\right] \rightarrow 0,
$$

$$
\frac{1}{C} \sum_{c=1}^{C} I\left[\sqrt{n \cdot 2}\left(\check{m}_{1 n \cdot 2}^{+\star c}-\check{m}_{1 n \cdot 2}^{+}\right) \leq x\right]-P\left[\sqrt{n_{\cdot 2}}\left(\check{m}_{1 n \cdot 2}^{+}-m_{1 t r}^{+}\right) \leq x\right] \rightarrow 0,
$$

$$
\frac{1}{C} \sum_{c=1}^{C} I\left[\sqrt{n_{\cdot 2}}\left(m m_{1 n_{\cdot 2}}^{\star c}-\check{m} m_{1 n_{\cdot 2}}\right) \leq x\right]-P\left[\sqrt{n_{\cdot 2}}\left(\check{m}^{\prime} m_{1 n_{\cdot 2}}^{-}-m m_{1 t r}\right) \leq x\right] \rightarrow 0
$$
in probability as $n_{p 2} \rightarrow \infty$ and $C \rightarrow \infty$.

\section{NUMERICAL EXPERIMENTS}

We use data collected and analyzed by Håkansson (2007) as if they were obtained on the first step in our two-step design of data collecting. During the first step of data collecting randomly sampled $n_{1}=241$ respondents report self-selected WTP-intervals, containing their true WTPpoints. The set $\mathcal{U}_{m}=\left\{\mathbf{u}_{1}, \ldots, \mathbf{u}_{m}\right\}$ contains all intervals $\mathbf{u}_{h}=\left(u_{L h}, u_{R h}\right], h=$ $1, \ldots, m$. The following four WTP-intervals $(50,100],(20,50],(100,200]$, and $(20,100]$ have been stated 69, 39, 23, and 11 times respectively, i.e. near $60 \%$ of all respondents have picked these four intervals. The variability of
6

7

8 


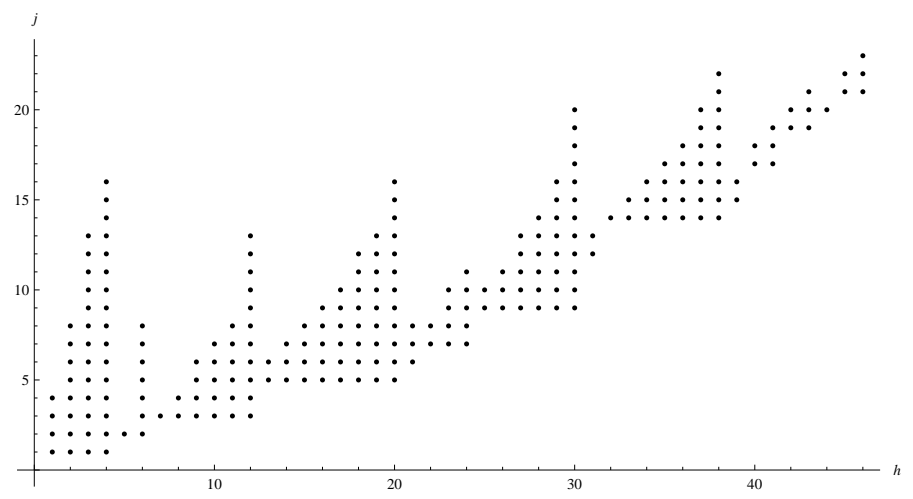

Figure 1.- The set of all compatible indexes $h, j, h=1, \ldots, 46, j=1, \ldots, 23$. The sets $\mathcal{C}_{h}=\left\{j, \mathbf{v}_{j} \subseteq \mathbf{u}_{h}\right\}, \mathcal{D}_{j}=\left\{h, \mathbf{v}_{j} \subseteq \mathbf{u}_{h}\right\}$, are $h$-cuts and $j$-cuts of the shown set.

rounded self-selected intervals can be described by disjoint division intervals $\mathcal{V}_{k}=\left\{\mathbf{v}_{1}, \ldots, \mathbf{v}_{k}\right\}$ corresponding to $\mathcal{U}_{m}$. In our numerical experiment we have the following, corresponding to $U_{m}, m=46$, division intervals: $v_{1}=(0,5], v_{2}=(5,10], v_{3}=(10,15], v_{4}=(15,20], v_{5}=(20,25], v_{6}=$ $(25,30], v_{7}=(30,40], v_{8}=(40,50], v_{9}=(50,60], v_{10}=(60,70], v_{11}=(70,75]$, $v_{12}=(75,80], v_{13}=(80,100], v_{14}=(100,150], v_{15}=(150,170], v_{16}=(170,200]$, $v_{17}=(200,250], v_{18}=(250,300], v_{19}=(300,400], v_{20}=(400,500], v_{21}=$ $(500,600], v_{22}=(600,1000], v_{23}=(1000,2000]$.

Each $\mathbf{u}_{h} \in \mathcal{U}_{m}$ is the union of related division intervals. Pairs of indexes $\{h, j\}$ are compatible if $\mathbf{v}_{j} \subseteq \mathbf{u}_{h}$. We order the indexes as follows: $h_{1}<h_{2}$ if $u_{L h_{1}}<u_{L h_{2}}$ or if $u_{L h_{1}}=u_{L h_{2}}$ and $u_{R h_{1}}<u_{R h_{2}}$, and then let $j_{1}<j_{2}$ if $v_{L j_{1}}<v_{L j_{2}}$. The set of all compatible indexes $\{h, j\}$ is shown in Figure 1. The sets of indexes $\mathcal{C}_{h}=\left\{j: \mathbf{v}_{j} \subseteq \mathbf{u}_{h}\right\}$ and $\mathcal{D}_{j}=\left\{h: \mathbf{v}_{j} \subseteq \mathbf{u}_{h}\right\}$, are the vertical cuts of the set with fixed $h$, and the horizontal cuts with fixed $j$, respectively. $n_{1}=241$ intervals have been collected and 21 intervals have been stated only once.

From (3.1) we estimate the coverage probability by $\hat{p}_{c}[241]=0.9128$. In 


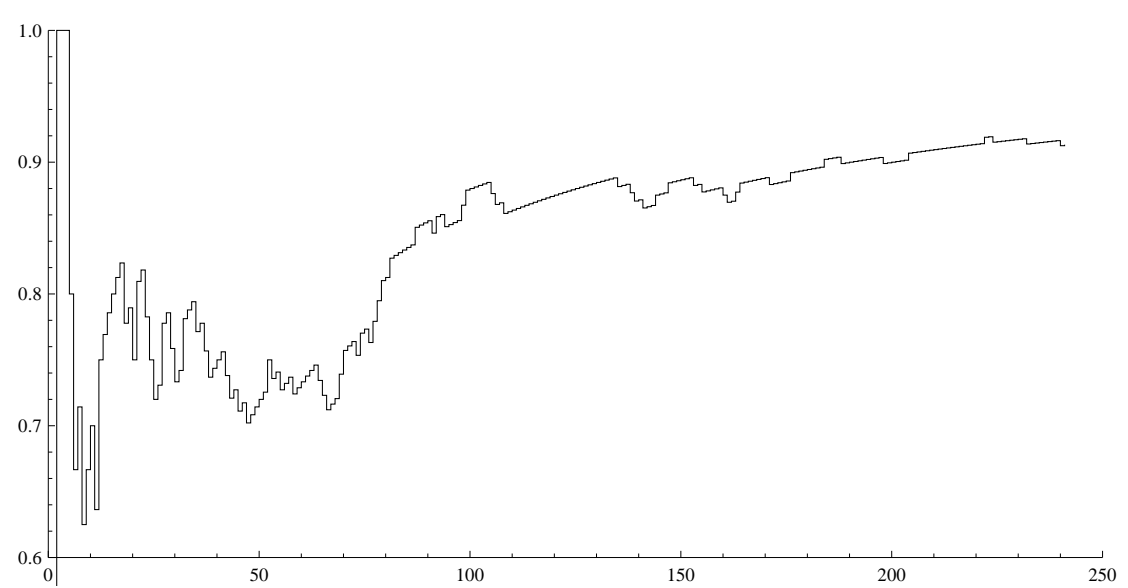

FIGURE 2.- The dynamics of the coverage probability estimates $\hat{p}_{c}[i]$ as a function of $i$ respondents sequentially selected on the first step.

Figure 2 the part of the plot, with values $\hat{p}_{c}[i], i=1,2, \ldots, 241$, is shown. The coverage probability $\hat{p}_{c}[i]$ characterizes the fraction of respondents in the whole population, who being selected after the $i$ th respondent, that happen to communicate an interval that has been chosen among the first $i$ selected respondents. A more detailed evolution of $\hat{p}_{c}[i], 180 \leq i \leq 241$ is shown in Figure 3.

If we knew all conditional probabilities $w_{h j}=P\left[H_{i}=h \mid X_{i} \in \mathbf{v}_{j}\right]$ then we could consistently estimate many useful characteristics of the true WTPdistribution using only WTP-intervals in the first step. If we know $\mathbb{W}_{m k}=$ $\left(w_{h j}\right)$ then under the Assumptions 1 - 3 we can write the log likelihood normed by $n_{1}$ as follows

$$
\operatorname{lik}\left[\mathbf{q}_{k} \mid \mathbb{W}_{m k}, t_{1}, \ldots, t_{m}\right]=\sum_{h=1}^{m} \frac{t_{h}}{n_{1}} \log \left[\sum_{j \in \mathfrak{C}_{h}} w_{h j} q_{j}\right], q_{j} \geq 0, \sum_{j=1}^{n} q_{j}=1 .
$$

One can consider many examples with different arrays $\mathbb{W}_{m k}$. Each $\mathbb{W}_{m k}$ describes a specific behavior of respondents in their selection of intervals. We 


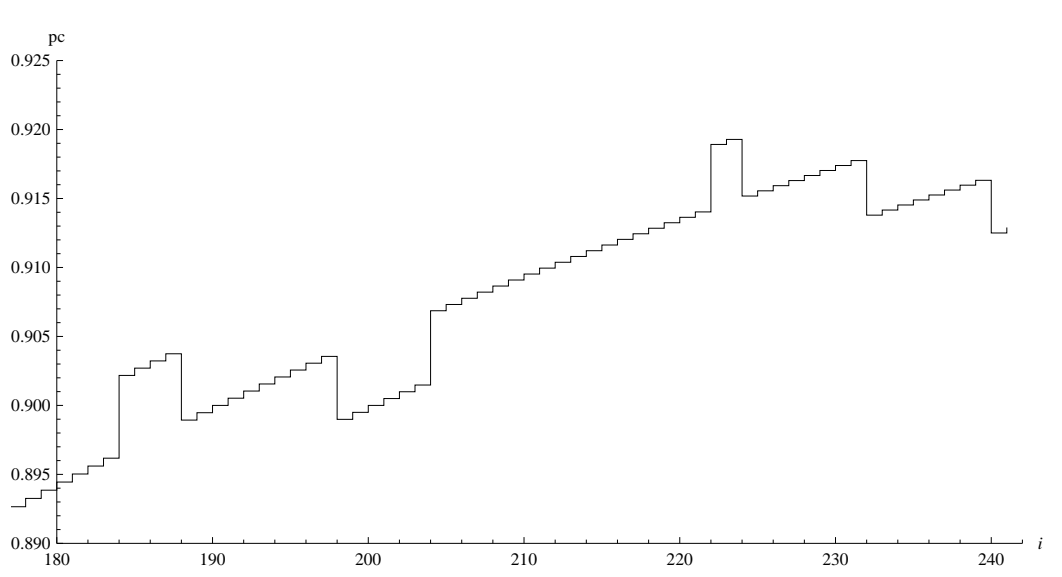

FIGURE 3.- The dynamic in $i$ of the probability $\hat{p}_{c}[i]$ for $i$ in $\{180, \ldots, 241\}$.

have considered the following five hypothetical behavior models of respondents:

BM1 is the behavior model of indifferent respondents with $w_{h j}=1 / d_{j}, h \in$ $\mathcal{D}_{j}, j=1, \ldots, k(=23), d_{j}$ is the size of $\mathcal{D}_{j}$.

BM2 is the behavior model of respondents who, with $\mathbf{v}_{j}$ containing their WTP-point, that chooses $\mathbf{u}_{h}$ in which $\mathbf{v}_{j}$ is the last division interval.

BM3 is the behavior model of respondents who, with $\mathbf{v}_{j}$ containing their WTP-point, pick $\mathbf{u}_{h}, h \in \mathcal{D}_{j}$ with probability proportional to $w_{t r h}, h=$ $1, \ldots, m$.

Here, by using estimated frequencies $\hat{w}_{h}=\frac{t_{h}}{n}$ we take

$$
w_{h j}=\frac{t_{h}}{\left(\sum_{h^{\prime} \in \mathcal{D}_{j}} t_{h^{\prime}}\right)} .
$$

If $\mathbf{u}_{h}=\cup_{j \in \mathcal{C}_{h}} \mathbf{v}_{j}=\mathbf{v}_{j_{h}+1}+\mathbf{v}_{j_{h}+2}+\cdots+\mathbf{v}_{j_{h}+M}$ then $\mathbf{v}_{j}=\mathbf{v}_{j_{h}+a} \subseteq \mathbf{u}_{h}$ has internal rank $r_{h j}=a \leq M$. The notion of the interval rank is used in two following behavior models.

BM4 is the behavior model of respondents who, with $\mathbf{v}_{j}$ containing their WTP-point, report $\mathbf{u}_{h}, h \in \mathcal{D}_{j}$, with probabilities proportional to 
$w_{h}\left(r_{h j} / r_{h M}\right)^{c 1-1}\left(1-\left(r_{h j} / r_{h M}\right)^{c 2-1}\right.$, i.e. with the discrete Beta-type weighting

BM5 is the behavior model of respondents who, with $\mathbf{v}_{j}$ containing their WTP-point, select $\mathbf{u}_{h}, h \in \mathcal{D}_{j}$, with probability proportional to of probabilities $w_{h}, h=1, \ldots, m$.

Let $\operatorname{mllik}^{(B M)}$ be the maximum of llik in (5.1) with $\mathbb{W}_{m k}=\left(w_{h j}\right)$ corresponding behavior model $B M$. We can interpret the maxima of llik functions corresponding to different behavior models as measures of their plausibility to approximate real behavior of respondents. Note that $-\mathrm{mllik}^{(B M)}$ is the entropy of the distribution $\left\{\hat{w}_{1}^{(B M)}, \ldots, \hat{w}_{m}^{(B M)}\right\}$ of frequencies $\hat{w}_{h}^{(B M)}=$ $\sum_{j \in \mathcal{D}_{j}} w_{h j}^{(B M)} \check{q}_{j}^{(B M)}, B M=B M 1, \ldots, B M 5$. Here $\check{q}_{j}^{(B M)}$ are the ML-estimates of $q_{t r j}, j=1, \ldots, k$. The empirical entropy eew $=\sum_{h=1}^{m} t_{h} / n_{1} \log \left[n_{1} / t_{h}\right]=$ 2.8224 , where $t_{h}, h=1, \ldots, m=46$, are the numbers of respondents who chose $\mathbf{u}_{h} \in \mathcal{U}_{m}$. In our calculations we have supposed that the true WTPsurvival function is a mixture of the Weibull and the Exponential survival functions with unknown parameters $p, a, b, m_{1}$, i.e. $s f_{W T P}[x]=p e^{-(x / a)^{b}}+$ $(1-p) e^{-x / m_{1}}$. Results of our calculations are collected in the following Table 1.

Table 1. Comparisons with the empirical entropy eew $=2.8224$.

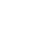




\begin{tabular}{|l|r|r|r|r|r|r|}
\hline$\frac{\text { Behavior }}{\text { Model }}$ & BM1 & BM2 & BM3 & BM4 & BM4 & BM5 \\
\hline $\mathrm{c} 1$ & - & - & - & 1.9256 & 1.9565 & 16.872 \\
\hline $\mathrm{c} 2$ & - & - & - & 1.5362 & 1.9565 & - \\
\hline $\mathrm{p}$ & 0.8849 & 0.7766 & 0.8512 & 0.8305 & 0.8426 & 0.8160 \\
\hline $\mathrm{a}$ & 77.4627 & 4.4643 & 74.0964 & 76.7255 & 74.7109 & 74.9982 \\
\hline $\mathrm{b}$ & 1.8425 & 1.9342 & 1.7638 & 1.8547 & 1.7942 & 1.8374 \\
\hline $\mathrm{m} 1$ & 286.7622 & 276.6101 & 259.3601 & 272.9070 & 271.5213 & 254.7344 \\
\hline mean WTP & 93.8918 & 126.854 & 94.7380 & 102.8351 & 98.73626 & 101.2456 \\
\hline mllik $^{(B M)}$ & -3.9388 & -3.4576 & -2.9301 & -2.9079 & -2.9109 & -2.8591 \\
\hline - $\frac{\text { mllik }^{(B M)}}{\text { eew }}$ & 1.396 & 1.225 & 1.038 & 1.030 & 1.031 & 1.013 \\
\hline
\end{tabular}

We see that the values of $\frac{\text { mllik }^{(B M)}}{e e w}$ depends on the behavior model $B M$. The

best correspondence we have is with $B M=B M 5$. This dependency on the assumed behavioral model shows why the direct usage of the survival theory, with the assumption of independency of censoring intervals from positions of covered true WTP-points, will lead to erroneous estimation of the true WTP-distribution. From Table 1 we conclude that to estimate conditional probabilities $\mathbb{W}_{m k}=\left(w_{h j}\right)$ consistently,we need extended empirical data. We can do that on the second step of data collecting.

In our numerical experiment we suppose that the WTP-distribution is a $p_{W E}$-mixture WE of the Weibull distribution $W(a, b)$ and the Exponential distribution $E\left(m_{1}\right)$ with parameters $p_{W E}=0.8160, a=74.8992, b=$ $1.8374, m 1=254.7344$,

$$
s f_{W T P}[x]=p_{W E} e^{-(x / a)^{b}}+\left(1-p_{W E}\right) e^{-x / m_{1}} .
$$

We use the behavior model $B M 3$ with array $\mathbb{W}_{m k}=\left(w_{h j}\right)$, for any $j=$ $1, \ldots, k$, and $h \in \mathcal{D}_{j}, w_{h j}=t_{h} /\left(\sum_{h^{\prime} \in \mathcal{D}_{j}} t_{h}\right)$. The numbers $t_{h}, h=1, \ldots, m$, are taken from $\mathbf{d} \mathbf{1}_{n \cdot 1}$ and $w_{h j}$ correspond to (5.2). We could take any other array with $w_{h j} \geq 0$, and $\sum_{h^{\prime} \in \mathcal{D}_{j}} w_{h j}=1$. 
Let $p_{N A}$ be the probability that a respondent states only a self-selected interval. $\left(1-p_{N A}\right)$ is the probability of finding a respondent that reports a self-selected interval and a division interval, both containing the true point. If $\mathbf{u}_{h}$ has already been reported then the only $\mathbf{u}_{h}$ interval compatible with $\mathbf{v}_{j} \in \mathcal{C}_{h}$ containing the true WTP-point $X_{i}$ can be added. In the our example we let $p_{N A}=2 / 3$. Then, in the mean, two of each three respondents will reject to add division intervals to their self-selected intervals. Recall that we use the parameters stated just before (5.3).

Let $q_{j}=P\left[X_{i} \in \mathbf{v}_{j}\right]=s f_{W T P}\left[v_{L j}\right]-s f_{W T P}\left[v_{R j}\right]$ where the survival function is given in (5.3). With probability $p_{N A} q_{j} w_{h j}$ the $i$-th respondent reports $\mathbf{u}_{h}$ and rejects to add a $\mathbf{v}_{j}$. Then the single triple $\left\{i, \mathbf{u}_{h}, N A\right\}$ is added to simulated data. With probability $\left(1-p_{N A}\right) q_{j} w_{h j}$ the $i$-th respondent states both $\mathbf{u}_{h}$ and $\mathbf{v}_{j}$. Then the triple $\left\{i, \mathbf{u}_{h}, \mathbf{v}_{j}\right\}$ is added to simulated data.

We have simulated data $\mathbf{d} \boldsymbol{2}_{n \cdot 2}, n_{\cdot 2}=9000$, containing $n_{s 2}=5856$ singles and $n_{p 2}=3144$ pairs. Henceforth we call $\mathbf{d} \boldsymbol{2}_{n_{.2}}$ the true data. As well we call the WTP-survival function in (5.3) and corresponding characteristics "true". By using values $\mathbf{u}_{h_{i}}, \mathbf{v}_{j_{i}}$ in all $n_{p 2}$ pairs we obtain estimates $\check{q}_{p j}$ as in (4.2), $j=1, \ldots, 23$. To improve these estimates we have to use all data $\mathbf{d} \boldsymbol{2}_{n_{\text {.2 }}}$ with pairs and singles. The improved ML-estimate $\check{\mathbf{q}}_{k M}$, corresponding to log likelihood (4.7), can be obtained by the recursion (4.8). After 10 iterations with $q_{j}^{(1)}=\check{q}_{p j}$ we obtain the following list with approximating $q_{t r j}$ values $\check{q}_{j}^{(10)}, j=1, \ldots, 23$,

$\{0.01267,0.01848,0.02284,0.03063,0.03495,0.04188,0.09621,0.09395$,

0.08782, 0.08755, 0.04739, 0.03548, 0.12409, 0.12905, 0.02921, 0.01713, 0.01930, 0.01355, 0.02429, 0.00750, 0.00804, 0.01347, 0.00451\}.

In the Figure 4 the histogram of approximated estimates $\check{q}_{j}^{(10)}$ of $\check{q}_{t r j}, j=$ $1, \ldots, 23$, is shown together with the true probability density of the true WTP-distribution (5.3). The histogram is shown over the part of range $0 \leq x \leq 600 S E K$. 


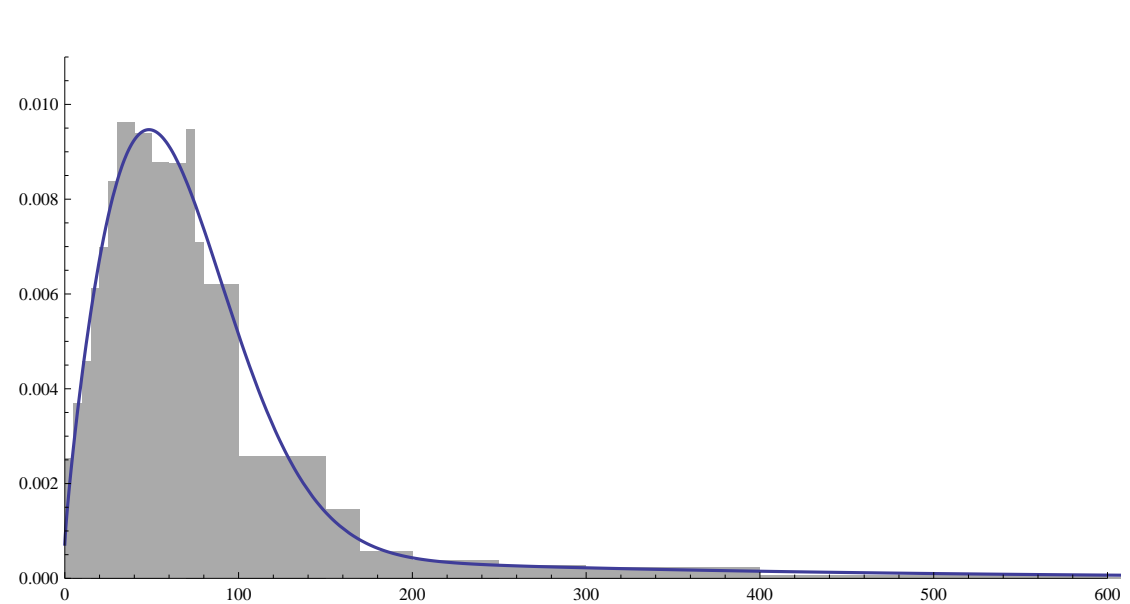

FiguRE 4.- The true probability density (smooth line) and the ML-estimate of the true WTP-histogram (light gray area) with stepwise boundary line.

Let $v_{R j}^{k}=\sum_{j^{\prime}=j}^{k} v_{R j^{\prime}}$ be values of the right divisions' ends, and let $q_{t r j}^{k}=$ $\sum_{j^{\prime}=1}^{k} q_{t r j^{\prime}}, \check{q}_{j}^{(10) k}=\sum_{j^{\prime}=1}^{k} \check{q}_{j^{\prime}}^{(10) k}$, where $\check{q}_{j}^{(10) k}$ are obtained after 10 iterations of recursion (4.8). The true points $\left\{v_{R j}^{k}, q_{t r j}^{k}\right\}$ on the true survival function (5.3) are approximated by points $\left\{v_{R j}^{k}, \check{q}_{j}^{(10)}\right\}$. The obtained approximation is rather accurate. To illustrate the result more clearly, Figure 5 shows the true WTP-survival function (5.3) together with its true points and approximated points $\left\{v_{R j}^{k}, \check{q}_{j}^{(10) k}\right\}$ over the part $0 \leq x \leq 200 S E K$ of the whole range $0 \leq x \leq 2000 S E K$. The area below the true WTP-survival function, shown in Figure 5 , is the true mean WTP-value $m_{1 t r}=101.161 S E K$. However, in an application we do not know the true WTP-survival function. As explained in Section 4 we may consider, as an approximation of $m_{1 t r}$, the WTP-medium mean value $m m_{1 t r}$. The true value is $m m_{1 t r}=103.718$. In our numerical experiment we can consistently estimate it as $n_{2} \rightarrow \infty$. By using 10 iteration with recursion (4.8) we obtain $q_{j}^{(10)}, j=1, \ldots, 23$. We substitute these values in (4.20) and obtain that the approximated ML-estimate $m m_{1}^{(10)}=104.281 S E K$. The deviations rmm $_{1}^{(10)}-m m_{1 t r}=0.563 S E K$, 


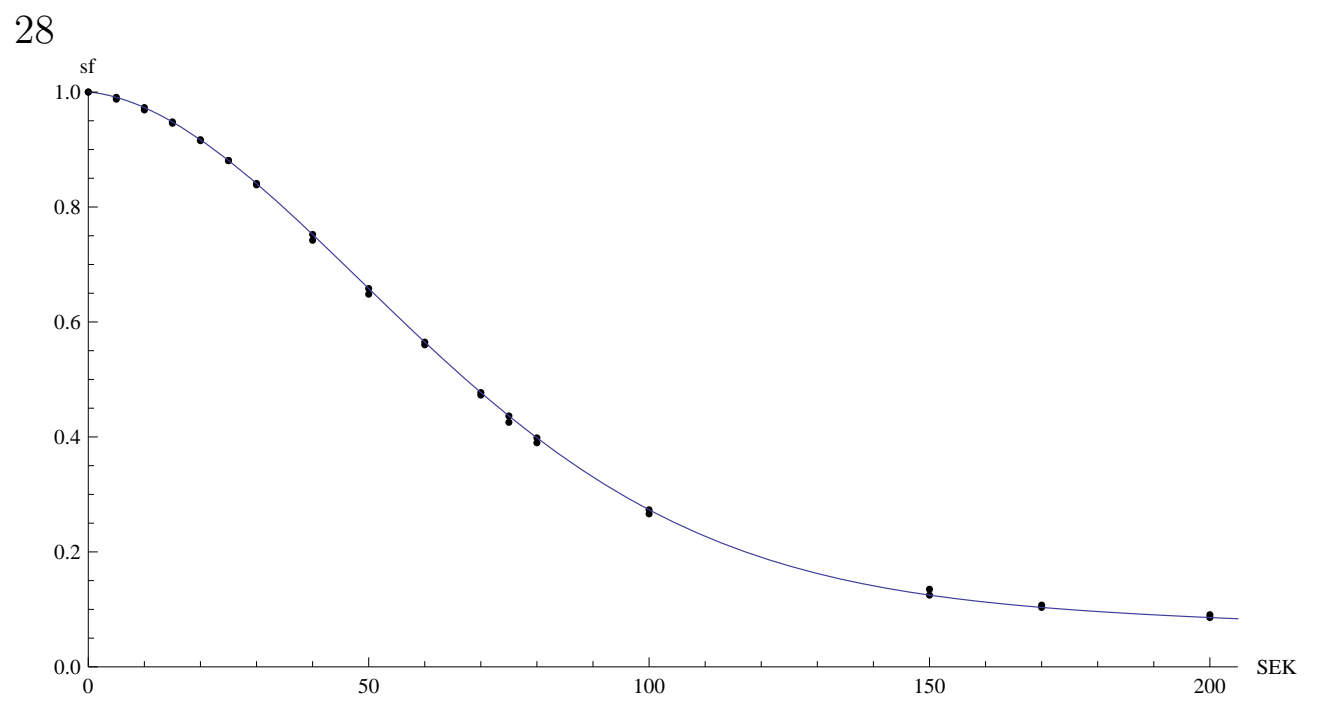

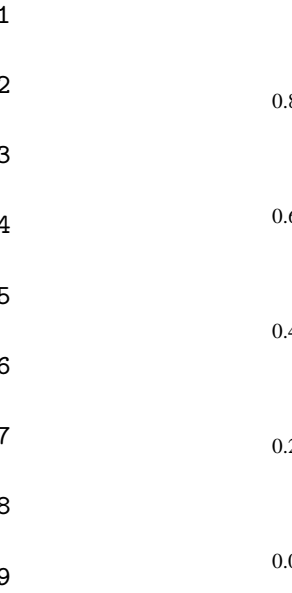

Figure 5.- The true WTP-survival function (5.3) is shown over interval $[0,200]$ together with its true points $\left\{v_{R j}^{k}, q_{t r j}^{k}\right\}$ and the approximated MLestimated points $\left\{v_{R j}^{k}, \check{q}_{j}^{(10) k}\right\}, 1 \leq j \leq 16$.

while the bias $m m_{1 t r}^{(10)}-m_{1 t r}=3.120 S E K$. Of course in reality we do not know the bias and these deviations. The bias would be smaller if, in the second step, the two subintervals $(100,120]$ and $(120,150]$ were used as the division intervals, rather than $v_{15}=(100,150]$. Note that the estimate $m^{(10)}$ is the area below the broken line joining neighbor points $\left\{v_{R j}^{k}, \check{q}_{j}^{(10) k}\right\}, j=1, \ldots, k, k=23$. The broken line is shown in Figure 6 .

Below we consider resampling methods to evaluation accuracies of MLestimators. In our numerical experiment we know all necessary parameters for simulation any number of data independent copies $\mathbf{d} \mathbf{2}_{n \cdot 2}^{c}, c=$ $1,2, \ldots, C$ which have the distribution identical with the considered above data $\mathbf{d} 2_{n \cdot 2}^{c}, n_{\cdot 2}=9000$. In our numerical experiment we simulate $C=2000$ such i.i.d. copies. Each simulated copy contains 9000 triplets (singles or pairs). Here the total collected data may be thought of as registered answers of 18 millions of respondents.

For each copy of data $\mathbf{d} \mathbf{2}_{n .2}^{c}$ we apply 5 iterations of the recursion (4.8) and 
1

2

3

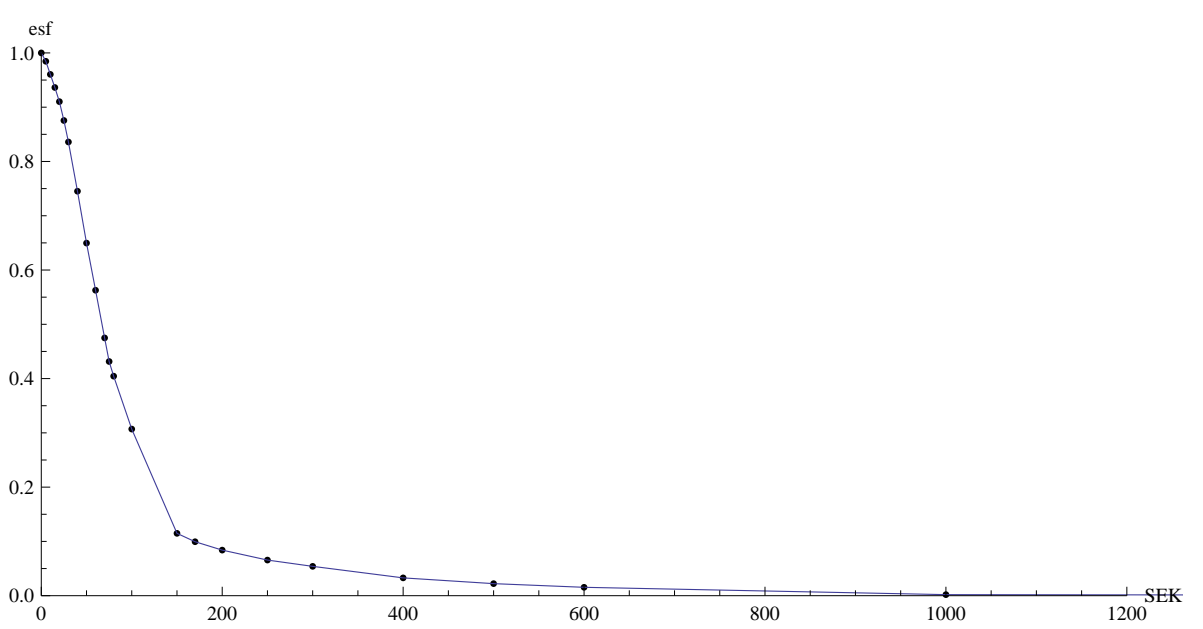

FiguRE 6.- The broken line joins by intervals the ML-estimates of division points of WTP-survival function.

obtain sufficiently accurate approximation $\check{\mathbf{q}}_{k}^{c(5)}$ of the ML-estimate $\check{\mathbf{q}}_{k M}^{c}$. Here, we use all copies of data, i.e. both pairs and singles. We can also find the ML-estimates $\check{\mathbf{q}}_{p k}^{c}$ of $\mathbf{q}_{t r k}$ by using (4.2) and the part of data $\mathbf{d} \mathbf{2}_{n \cdot 2}^{c}$ with triples containing pairs. By substituting $\check{\mathbf{q}}_{p k}^{c}$ and $\check{\mathbf{q}}_{k}^{c(5)}$ in (4.21) we obtain estimates of WTP-medium mean values $\check{m m}_{1 p}^{c}$ and $m m_{1}^{c(5)}, c=1, \ldots, R$. To underline the gain of usage the whole data $\mathbf{d} \boldsymbol{2}_{n \cdot 2}^{c}$ compared with only the part of all pairs we calculate two lists with $C=2000$ deviations

$$
\check{d e} v_{1}^{c(5)}=m m_{1}^{c(5)}-m m_{1 t r}
$$

$$
\check{d e v}_{1 p}^{c}=m \check{m}_{1 p}^{c}-m m_{1 t r}, \quad c=1, \ldots, C .
$$

The deviations (5.4) and (5.5) can be considered as values $\left\{d \check{e} v_{1}^{c(5)}\right.$, ďev $\left.\check{e}_{1 p}^{c}\right\}$ of i.i.d. two-dimensional r.v.s. Then their standard deviation estimates are $\check{s t} d_{1}^{(5)}=\left(\frac{1}{R-1} \sum_{c=1}^{R}\left(\tilde{d e} v_{1}^{c(5)}\right)^{2}\right)^{1 / 2}=1.780$, $\check{s t} d_{1 p}=\left(\frac{1}{R-1} \sum_{c=1}^{k}\left(\check{d e} v_{1 p}^{c}\right)^{2}\right)^{1 / 2}=2.507$. 
It is useful to know the following empirical distribution functions (e.d.f. s)

$$
e \check{d f} f_{1 C}^{(5)}[x]=\frac{1}{C} \sum_{c=1}^{C} I\left[\check{d e} v_{1}^{c(5)} \leq x\right],
$$

$$
e \check{d} f_{1 p C}[x]=\frac{1}{C} \sum_{c=1}^{C} I\left[\check{d e}_{1 p}^{c} \leq x\right] .
$$

In Appendix we prove that the consistent ML-estimate of $\mathbf{q}_{t r k}$ exists and all regularity properties RP1 - RP5 are valid. Then we may apply resampling methods which will give us consistent estimate of characteristics of deviations (5.4) - (5.7). We simulate $C=2000$ independent copies resampled data $\mathbf{d} \boldsymbol{2}_{n .2}^{\star c}, c=1, \ldots, C$. Then we calculate corresponding estimates of WTP-medium mean values $m m_{1}^{\star c(5)}$ and $m m_{1 p}^{\star c}$ similarly as above using $\mathbf{d} \boldsymbol{2}_{n \cdot 2}^{\star c}$ instead of the true data $\mathbf{d} \boldsymbol{2}_{n \cdot 2}$ and five iterations by the recursion (4.14). Here we also use the approximated ML-estimate $m m_{1}^{(5)}$ based on true data $\mathbf{d} \boldsymbol{2}_{n \cdot 2}$. The deviations $m \check{m} m_{1}^{\star c(5)}$ and $m \check{m} m_{1 p}^{\star c}$ from the ML-estimates of $m m_{1 t r}$ based on true whole data $\mathbf{d} \mathbf{2}_{n \cdot 2}$ and on its part with pairs are

$$
\check{d e} v_{1}^{\star c(5)}=\operatorname{mim}_{1}^{\star c(5)}-\check{m m_{1}^{(5)}}
$$

$$
\check{d e v_{1 p}^{\star c}}=\check{m m_{1}^{\star c}}-\check{m m_{1 p}} .
$$

These deviations are used in the following e.d.f.s

$$
e_{e} f_{1 C}^{\star(5)}[x]=\frac{1}{C} \sum_{c=1}^{C} I\left[\check{d e} v_{1}^{\star c(5)} \leq x\right]
$$

$$
e \check{d} f_{1 p C}^{\star}[x]=\frac{1}{C} \sum_{c=1}^{C} I\left[\check{d e} v_{1 p}^{\star c} \leq x\right] .
$$

These e.d.f.s (5.10) and (5.11) correspond to the usage of all triples in $\mathbf{d} \mathbf{2}_{n \cdot 2}^{\star c}$ and only triples with pairs in $\mathbf{d} \boldsymbol{2}_{n .2}^{\star c}$, respectively. 

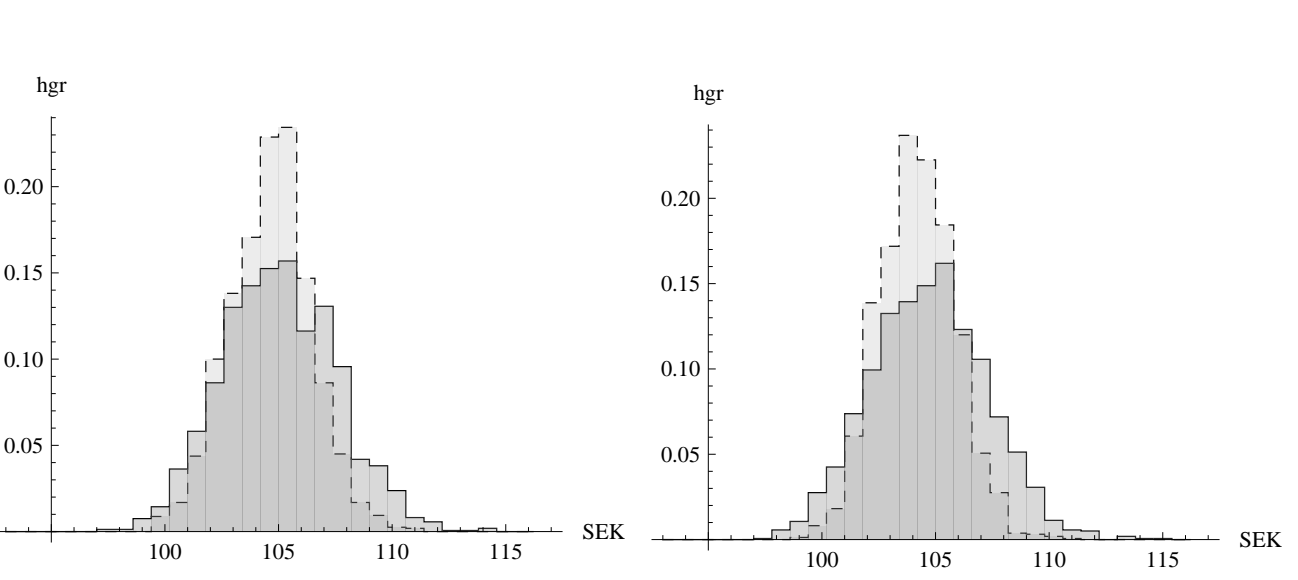

Figure 7.- Two pairs of histograms, corresponding to $C=2000$ deviations (5.4), (5.5) and (5.8), (5.9), are given in the left and right plots, respectively. Dashed contour lines are used for deviations (5.4) and (5.9) based on triples with both pairs and singles, otherwise for triples with only pairs solid contour lines are used.

The lists with deviations (5.4), (5.5) and (5.8), (5.9) can be presented as histograms, composed from rectangles with low edges intervals $I_{r}=$ $(2 r, 2(r+1)], r=0,1, \ldots$ on the $x$-axis. The length of $I_{r}$ is $2 S E K$. The rectangles side intervals are proportional to the numbers of $m^{\check{m}}{ }_{1}^{c(5)} \in I_{r}$ and $\mathrm{mm}_{1 p}^{c} \in I_{r}, c=1, \ldots, C$. Two corresponding histograms are in the left plot in Figure 7. The histograms corresponding to deviations (5.8) and (5.9) are in the right plot of Figure 7.

We may visualize these e.d.f.s (5.6) and (5.7) in a $Q Q$-plot with Normal quantiles. $Q Q$-plots are common in statistical applications because they give essential information about deviations of e.d.f.s of interest from corresponding limit distributions as size data is growing. The left $Q Q$-plot in Figure 8 contains two empirical distribution functions (5.6) and (5.7) (solid and dashed lines, respectively). We see that the empirical distributions (5.6) and (5.7), shown in the left $Q Q$-plot in Figure 8 are close to Normal. The 

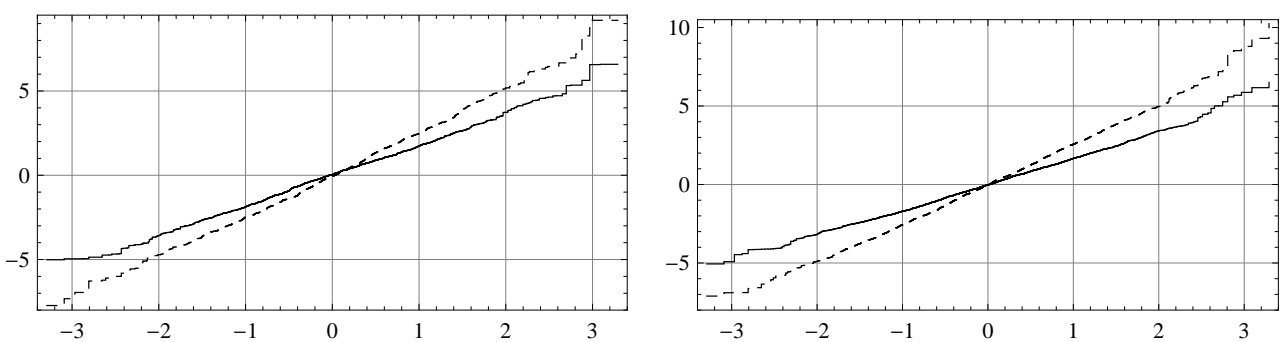

Figure 8.- Two $Q Q$-plots, with Normal quantiles, contain lines with empirical distribution functions of deviations of (5.6) and (5.7) in the left $Q Q$-plot and (5.10) and (5.11) in the right $Q Q$-plot. Solid lines correspond to usage copies of data with both pairs and singles. Dashed lines correspond to usage only copies of triples with pairs.

deviations, in the case when both triples with singles and pairs are used, are essentially less than if only triplets with pairs are used.

In the concluding Figure 9 we display two e.d.f.s (5.7) and (5.10). In a real application, the dashed line is not known and an estimate of it (solid line) is the main aim of the suggested approach based on resampling methods. Figure 9 illustrates the consistency of the suggested estimators.

\section{SUMMARY AND CONCLUSION}

We have proposed a natural extension of the self-selected intervals approach to information elicitation in surveys. Because a point is a special case of an interval, self-selected intervals are very general and an interesting candidate in many types of surveys. Interest is also on the rise, as witnessed e.g. by recent changes towards the use of self-selected intervals in the Michigan Health Survey. While a single self-selected interval provides much useful information about respondent sentiments, our analysis suggest that identification of non-parametric estimators is difficult, if not impossible. We solve this identification problem by adding more information in a second step. 


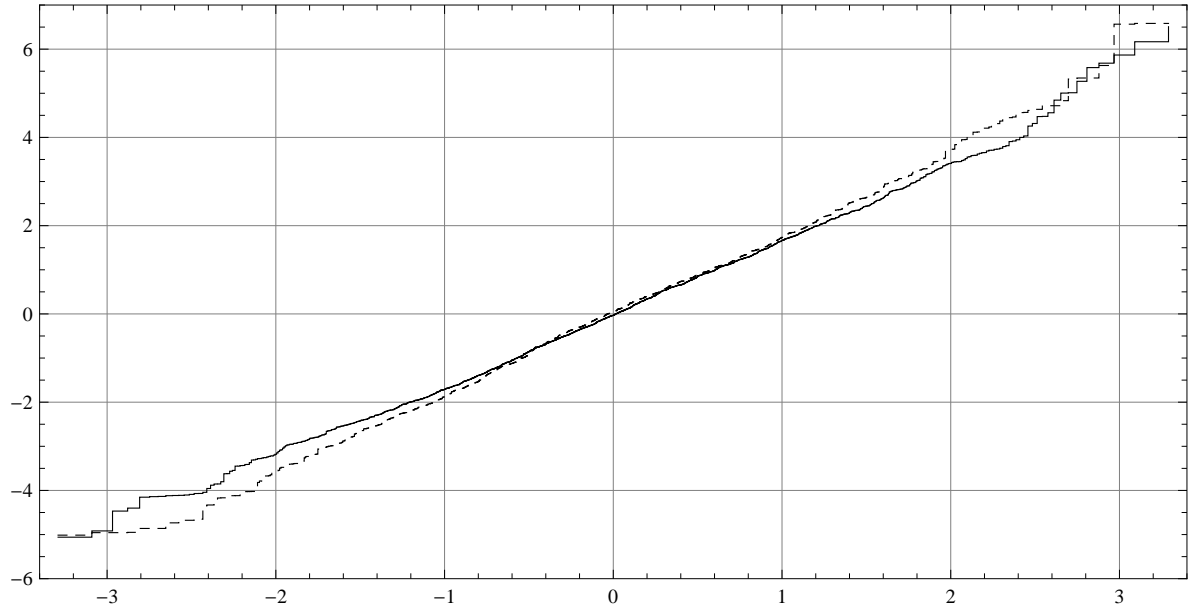

Figure 9.- This $Q Q$-plot shows how two distributions of the ML-estimates of medium mean WTP-values deviates from their average values. The dashed line corresponds the true distribution of the deviates based on $C=2000$ simulated copies of the true data $\mathbf{d} \mathbf{2}_{n .2}^{c}$ and on usage of the approximated ML-estimates of WTP-medium mean values based on WTP-probabilities $\left\{\check{q}_{1}^{(5)}, \ldots, \check{q}_{k}^{(5)}\right\}$. The solid line corresponds to the distribution of the similar deviates based on $C=2000$ resampled copies $\mathbf{d} \mathbf{2}_{n \cdot 2}^{\star c}$ of the list with original true data $\mathbf{d} \mathbf{2}_{n \cdot 2}$.

The granulation is based on a certain kind of division, where the division of the interval is based on the data. We also propose a stopping rule for sampling of respondents, which is quite practical. The investigator is to suggest a coverage probability, say 0.95 , which when reached means that there is a $5 \%$ chance that the next sampled self-selected interval will be different from the ones already stated. Theorem 1 proves that the proposed nonparametric MLE is consistent, Theorem 2 shows that the accuracy of the estimator can be consistently estimated by resampling. Thus, our proposed statistical model has a number of attractive properties.

The R-programs that have been developed should be useful in applying the suggested approach. The programs will be available on CRAN. Inter-

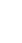

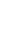

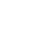

(3)


ested readers can also use an initially developed Mathematica version of the programs.

Our approach is currently silent about how to add background variables (barring a simple stratification of the data). This is work in progress. Overall, we would be very grateful for any comments or suggestions.

\section{APPENDIX}

We consider an approach to obtaining consistent estimators of the probabilities $q_{t r j}=P\left[X_{i} \in \mathbf{v}_{j}\right], \mathbf{v}_{j} \in \mathcal{V}_{k}, j=1, \ldots, k$, by maximizing the log likelihood function (4.7). The vector, of true parameters $\mathbf{q}_{t r k}=\left\{q_{t r 1}, \ldots, q_{t r k}\right\}$, is a point in the $(k-1)$-dimensional polyhedron $\mathcal{S}_{k-1}$ defined in (4.4). Remind that we consider the case with all $q_{t r j}>0, j=1, \ldots, k$. The set

$$
\breve{\mathcal{S}}_{k-1}=\left\{\mathbf{q}_{k}=\left\{q_{1}, \ldots, q_{k}\right\}: 0<q_{j}<1, \sum_{j=1}^{k} q_{j}=1\right.
$$

is the open convex set of all inner points of $\mathcal{S}_{k-1}$. The set with boundary points $\partial \mathcal{S}_{k-1}=\mathfrak{S}_{k-1} \backslash \breve{\mathcal{S}}_{k-1}=\left\{\mathbf{q}_{k}=\left\{q_{1}, \ldots, q_{k}\right\}: \min _{1 \leq j \leq k} q_{j}=0, \sum_{j=1}^{k} q_{j}=\right.$ $1\}$. We say that $n_{2}$ is sufficiently large if in the collected on the second step data $\mathbf{d} 2_{n \cdot 2}$ all $t_{p j}>0, t_{s h}>0, j=1, \ldots, k, h=1, \ldots, m$.

THEOREM 7.1 For every sufficiently large $n_{.2}$ the log likelihood (4.7) is concave on $\breve{S}_{k-1}$.

Proof. Let us take any two different points $\mathbf{q}_{k i} \in \breve{\mathcal{S}}_{k-1}, i=1,2$. Then all points

$$
\mathbf{q}_{k}[t]=(1-t) \mathbf{q}_{k 1}+t \mathbf{q}_{k 2} \in \breve{\mathcal{S}}_{k-1}, \quad 0 \leq t \leq 1,
$$

constitute the interval $\mathcal{J}\left[\mathbf{q}_{k 1}, \mathbf{q}_{k 2}\right] \subset \breve{S}_{k-1}$ connecting these two points. Let $f_{j}[t]=\log \left[q_{j}[t]\right], j=1, \ldots, k, g_{h}[t]=\log \left[\sum_{j \in \mathcal{C}_{h}} \hat{w}_{h j} q_{j}[t]\right], h=1, \ldots, m$. Their second derivatives are

$$
\frac{d^{2}}{d t^{2}} f_{j}[t]=-\frac{\left(q_{j 2}-q_{j 1}\right)^{2}}{q_{j}[t]^{2}}, \quad \frac{d^{2}}{d t^{2}} g_{h}[t]=-\frac{\left(\sum_{j \in \mathcal{C}_{h}} \hat{w}_{h j}\left(q_{j 2}-q_{j 1}\right)\right)^{2}}{\left(\sum_{j \in \mathcal{C}_{h}} \hat{w}_{h j} q_{j}[t]\right)^{2}} .
$$


From (7.2) it follows that the second sum in (4.7) has negative second derivative

$$
\frac{d^{2}}{d t^{2}}\left(\frac{n_{p 2}}{n_{\cdot 2}} \sum_{j=1}^{k} \frac{c_{p j}}{n_{p 2}} \log \left[q_{j}[t]\right]\right)<0, \quad 0 \leq t \leq 1
$$

1

2

3

Similarly, we obtain that the second derivative, of the first sum in (4.7), is non-positive

$$
\frac{d^{2}}{d t^{2}}\left(\frac{n_{s 2}}{n_{\cdot 2}} \sum_{h=1}^{m} \frac{t_{s h}}{n_{s 2}} \log \left[\sum_{j \in \mathfrak{C}_{h}} \hat{w}_{h j} q_{j}[t]\right]\right) \leq 0, \quad 0 \leq t \leq 1
$$

The second derivative of the last sum in (4.7) is zero. The second derivative of the log likelihood (4.7) is the sum of (7.3) and (7.4). Therefore, the second derivative is negative along intervals with any terminal points $\mathbf{q}_{k 1}, \mathbf{q}_{k 2}$ in $\breve{\mathcal{S}}_{k-1}$ defined in (7.1). Hence, the log likelihood (4.7) is concave on $\breve{\mathcal{S}}_{k-1}$.

Corollary 7.1 The log likelihood (4.7) attains its maximum at a point $\check{\mathbf{q}}_{k M}=\left\{\check{q}_{1 M}, \ldots, \check{q}_{k M}\right\} \in \breve{\mathcal{S}}_{k-1}$ and $\check{\mathbf{q}}_{k M}$ is the unique stationary point of log likelihood (4.7) in $\breve{\mathcal{S}}_{k-1}$.

Proof. Let us write in short $\operatorname{llik}\left[\mathbf{q}_{k}\right]=\operatorname{llik}\left[\mathbf{q}_{k} \mid \mathbb{W}_{m k}, \mathbf{d} \mathbf{2}_{n_{\cdot 2}}\right], \mathbf{q}_{k}=\left\{q_{1}, \ldots, q_{k}\right\}$. The function $\operatorname{llik}\left[\mathbf{q}_{k}\right]<0$ at any $\mathbf{q}_{k}$ in $\breve{\mathcal{S}}_{k-1}$. Let $\mathbf{q}_{k 0}$ be a point in $\breve{\mathcal{S}}_{k-1}$, $l_{0}=\operatorname{llik}\left[\mathbf{q}_{k 0}\right]$ and $Q\left(\mathbf{q}_{k 0}\right)=\left\{\mathbf{q}_{k}: \operatorname{llik}\left[\mathbf{q}_{k}\right] \geq l_{0}, \mathbf{q}_{k} \in \breve{S}_{k-1}\right\}$. From concavity of llik $\left[\mathbf{q}_{k}\right]$ function on $\breve{\mathcal{S}}_{k-1}$ it follows that $Q\left(\mathbf{q}_{k 0}\right)$ is convex and compact. The function $\operatorname{llik}\left[\mathbf{q}_{k}\right]$ is continuous on $Q\left(\mathbf{q}_{k 0}\right)$. If $\mathbf{q}_{k} \in \breve{\mathcal{S}}_{k-1} \backslash Q\left(\mathbf{q}_{k 0}\right)$ then $\operatorname{llik}\left[\mathbf{q}_{k}\right]<l_{0}$. Hence, the global maximum of $\operatorname{llik}\left[\mathbf{q}_{k}\right]$ on $\breve{\mathcal{S}}_{k-1}$ is attained on $Q\left(\mathbf{q}_{k 0}\right)$. From concavity of $\operatorname{llik}\left[\mathbf{q}_{k}\right]$ on $Q\left(\mathbf{q}_{k 0}\right)$ it follows that the point $\check{\mathbf{q}}_{k M}=\check{\mathbf{q}}_{k M}\left(n_{\cdot 2}\right)$, where $\operatorname{llik}\left[\check{\mathbf{q}}_{k M}\right]=\max _{\mathbf{q}_{k} \in Q\left[q_{k 0}\right]} \operatorname{llik}\left[\mathbf{q}_{k}\right]$, is unique, and all first order partial derivatives $\left.\frac{\partial \operatorname{llik}\left[\mathbf{q}_{k}\right]}{\partial q_{j}}\right|_{\mathbf{q}_{k}=\check{\mathbf{q}}_{k M}}=0, j=1, \ldots, k$. Hence, $\check{\mathbf{q}}_{k M}$ is the unique stationary point of $\operatorname{llik}\left[\mathbf{q}_{k}\right]$ in $\breve{\mathcal{S}}_{k-1}$. 
Now, we want to find a convenient method for search of the stationary point $\check{\mathbf{q}}_{k M}$ corresponding to the maximum value of (4.7) in the multidimensional set $\breve{\mathcal{S}}_{k-1}$. We will use the method of Lagrange multipliers, see e.g. Brinkhuis and Tihomirov (2005). We introduce an auxiliary parameter $\lambda$ and consider the following Lagrange function

$$
\varphi_{L}\left[\mathbf{q}_{k}, \lambda\right]=\operatorname{lik}\left[\mathbf{q}_{k} \mid \hat{\mathbb{W}}_{m k}, \mathbf{d} \boldsymbol{2}_{n \cdot 2}\right]+\lambda\left(q_{1}+\cdots+q_{k}\right)
$$

The first order partial derivatives of (7.5) w.r.t. components in $\mathbf{q}_{k}$ at a stationary point in $\breve{\mathcal{S}}_{k-1}$, are zeros

$$
\frac{n_{s 2}}{n_{\cdot 2}} \sum_{h \in \mathcal{D}_{j}} \frac{t_{s h}}{n_{s 2}} \frac{\hat{w}_{h j}}{\sum_{j^{\prime} \in \mathfrak{C}_{h}} \hat{w}_{h j^{\prime}} q_{j^{\prime}}}+\frac{n_{p 2}}{n_{\cdot 2}} \frac{t_{p j}}{n_{p 2}} \frac{1}{q_{j}}+\lambda=0, \quad j=1, \ldots, k .
$$

Note that

$$
\sum_{h=1}^{m} \frac{t_{s h}}{n_{s 2}}=\sum_{j=1}^{k} \frac{t_{p j}}{n_{p 2}}=1
$$

$$
\sum_{j=1}^{k}\left(\sum_{h \in \mathcal{D}_{j}} \frac{t_{s h}}{n_{s 2}} \frac{\hat{w}_{h j} q_{j}}{\sum_{j^{\prime} \in \mathcal{C}_{h}} \hat{w}_{h j^{\prime}} q_{j^{\prime}}}\right)=\sum_{h=1}^{m} \frac{t_{s h}}{n_{s 2}} \sum_{j \in \mathfrak{C}_{h}}\left(\frac{\hat{w}_{h j} q_{j}}{\sum_{j^{\prime} \in \mathfrak{C}_{h}} \hat{w}_{h j^{\prime}} q_{j^{\prime}}}\right)=1
$$

Then from (7.7), (7.8) by taking sum of (7.6), multiplied by $q_{j}$, over $j=$ $1, \ldots, k$, it follows that $\lambda=-1$. Hence, each of $k$ equalities (7.6) can be rewritten as follows

$$
q_{j}=\frac{n_{p 2}}{n_{\cdot 2}} \frac{t_{p j}}{n_{p 2}}+\frac{n_{s 2}}{n_{\cdot 2}} \sum_{h \in \mathcal{D}_{j}} \frac{t_{s h}}{n_{s 2}} \frac{\hat{w}_{h j} q_{j}}{\sum_{j^{\prime} \in \mathfrak{e}_{h}} \hat{w}_{h j^{\prime}} q_{j^{\prime}}}, j=1, \ldots, k .
$$

If $q_{1}, \ldots, q_{k}$ are solutions of (7.9) then all first order partial derivatives $\frac{\partial l i k\left[\mathbf{q}_{k}\right]}{\partial q_{j}}=0, j=1, \ldots, k$, and $\mathbf{q}_{k}=\left\{q_{1}, \ldots, q_{k}\right\}$ is a stationary point. From Corollary 7.1 we have $\mathbf{q}_{k}=\check{\mathbf{q}}_{k M}$.

From (7.9) one may suggest the following recursion with $g_{j}^{(1)}=t_{p j} / n_{p j}$

$$
q_{j}^{(r+1)}=\frac{n_{p 2}}{n_{\cdot 2}} q_{j}^{(1)}+\frac{n_{s 2}}{n_{\cdot 2}} \sum_{h \in \mathcal{D}_{j}} \frac{t_{s h}}{n_{s 2}} \frac{\hat{w}_{h j} q_{j}^{(r)}}{\sum_{j^{\prime} \in \mathfrak{C}_{h}} \hat{w}_{h j^{\prime}} q_{j^{\prime}}^{(r)}}, \quad r=1,2, \ldots
$$


COROllary 7.2 For any sufficiently large $n_{.2}$ recursion (7.10) generate the sequence of points $\mathbf{q}_{k}^{(r)}=\left\{q_{1}^{(r)}, \ldots, q_{k}^{(r)}\right\} \in \breve{S}_{k-1}, r=1,2, \ldots$, which converge to the unique stationary point $\check{\mathbf{q}}_{k M}=\left\{\check{q}_{1 M}, \ldots, \check{q}_{k M}\right\}$.

2

Proof. We can take as a start point $\mathbf{q}_{k}^{(1)}$ with $q_{j}^{(1)}=\frac{t_{p j}}{n_{p 2}}, j=1, \ldots, k$. We have

$$
\begin{aligned}
& q_{j}^{(1)} \rightarrow q_{t r j}>0, \quad \hat{w}_{h j} \rightarrow w_{h j}=P\left[H_{i}=h \mid X_{i}=x \in \mathbf{v}_{j}\right], \\
& \sum_{j^{\prime} \in \mathfrak{C}_{h}} \hat{w}_{h j} q_{j}^{(1)} \rightarrow w_{t r h}=\sum_{j \in \mathcal{C}_{h}} w_{h j} q_{t r j}>0, \quad \text { a.s. }
\end{aligned}
$$

as $n_{\cdot 2} \rightarrow \infty$. From (7.10) we have $q_{j}^{(r)} \geq \frac{n_{p 2}}{n \cdot 2} q_{j}^{(1)}>\frac{1}{2} \frac{n_{p 2}}{n_{.2}} q_{t r j}>0$ a.s. for all sufficiently large $n_{\cdot 2}$. From (7.7), (7.8), (7.11) and (7.10) it follows

$$
\sum_{j=1}^{k} q_{j}^{(r+1)}=\frac{n_{p 2}}{n_{\cdot 2}} \sum_{j=1}^{k} q_{j}^{(1)}+\frac{n_{s 2}}{n_{\cdot 2}} \sum_{j=1}^{k} \sum_{h \in \mathcal{D}_{j}} \frac{t_{s h}}{n_{s 2}} \frac{\hat{w}_{h j} q_{j}^{(r)}}{\sum_{j^{\prime} \in \mathfrak{C}_{h}} \hat{w}_{h j^{\prime}} q_{j}^{(r)}}=1
$$

If in (7.12) we start with $r=1$, then $\sum_{j=1}^{k} q_{j}^{(2)}=1$. We can apply the same argument with $r=2$ in (7.12). Then we obtain $\sum_{j=1}^{k} q_{j}^{(3)}=1$. Similarly we have $\sum_{j=1}^{k} q_{j}^{(r+1)}=1$ if $\sum_{j=1}^{k} q_{j}^{(r)}=1$. From (7.11) it follows that for any sufficiently large $n_{\cdot 2}$, and $r \geq 1, \mathbf{q}_{k}^{(r)} \in \mathcal{S}_{k-1}\left(\mathbf{q}_{t r k}\right)=\left\{\mathbf{q}_{k}: q_{j} \geq\right.$ $\left.\frac{1}{2} q_{t r j}, \sum_{j=1}^{k} q_{j}=1\right\}$ which is a compact set. Hence, there exists a limit point $\mathbf{q}_{k}^{(\infty)}, q_{j}^{(r)} \rightarrow q_{j}^{(\infty)}$, and $q_{k}^{(r+1)}-q_{k}^{(r)} \rightarrow 0, j=1, \ldots, k$, a.s. as $r \rightarrow \infty$. The partial derivatives $\frac{\partial \operatorname{llik}\left[\mathbf{q}_{k}\right]}{\partial q_{j}}, j=1, \ldots, k$, are uniformly continuous on $\mathcal{S}_{k-1}\left(\mathbf{q}_{t r k}\right)$. Then from (7.10) if $n_{\cdot 2}$ is sufficiently large we have that $\frac{\partial \operatorname{llik}\left[\mathbf{q}_{k}^{(r)}\right]}{\partial q_{j}} \rightarrow 0$ as $r \rightarrow \infty$. It follows that the limit point $\mathbf{q}_{k}^{(\infty)}$ is a stationary point. Due to concavity of (4.7) the stationary point $\mathbf{q}_{k}^{(\infty)}$ is unique, Therefore, from Corollary 7.1 it follows $\mathbf{q}_{k}^{(\infty)}=\check{\mathbf{q}}_{k M}$ and we have $q_{j}^{(r)} \rightarrow \check{q}_{j M}, j=1, \ldots, k$, as $r \rightarrow \infty$, for any sufficiently large $n_{\cdot 2}$.

If $n_{.2} \rightarrow \infty$ then all below following estimates a.s. converge to their limits

$$
\begin{aligned}
& \frac{n_{p 2}}{n_{\cdot 2}} \rightarrow \alpha_{p 2}>0, \quad \frac{n_{s 2}}{n_{\cdot 2}} \rightarrow \alpha_{s 2}>0, \quad \alpha_{p 2}+\alpha_{s 2}=1, \\
& \frac{t_{s h}}{n_{s 2}} \rightarrow w_{t r h}, \quad \frac{t_{p j}}{n_{p 2}} \rightarrow q_{t r j}, \quad \frac{t_{p h j}}{n_{p 2}} \rightarrow w_{h j} q_{t r j},
\end{aligned}
$$


$j=1, \ldots, k, h \in \mathcal{D}_{j}$. Then the $\log$ likelihood (4.7) on compact $\mathcal{S}_{k-1}\left(\mathbf{q}_{t r k}\right)$ uniformly converges, as $n_{\cdot 2} \rightarrow \infty$, to the non-random function of $\mathbf{q}_{k}$

$$
\operatorname{llik}^{(\infty)}\left[\mathbf{q}_{k}\right]=\alpha_{p 2} \sum_{j=1}^{k} q_{t r j} \log \left[q_{j}\right]+\alpha_{s 2} \sum_{h=1}^{m} w_{t r h} \log \left[\sum_{j \in \mathfrak{e}_{h}} w_{h j} q_{j}\right]+C_{p}
$$

where the constant $C_{p}=\alpha_{p 2} \sum_{j=1}^{k} \sum_{j \in \mathcal{D}_{j}} q_{t r j} \log \left[w_{h j}\right]$. In short we call $\operatorname{llik}^{(\infty)}\left[\mathrm{q}_{k}\right]$ as a limit log likelihood. The limit of concave functions (4.7) is above, by using the corresponding to (7.14) Lagrange function $\varphi_{L}^{(\infty)}\left(\mathbf{q}_{k}, \lambda\right)=$ $\operatorname{llik}^{(\infty)}\left[\mathbf{q}_{k}\right]+\lambda\left(q_{1}+\ldots+q_{k}\right)$, we find that the similar to (7.9) equations at the unique stationary point are

$$
q_{j}=\alpha_{p 2} q_{t r j}+\alpha_{s 2} \sum_{h \in \mathcal{D}_{j}} w_{t r h} \frac{w_{h j} q_{j}}{\sum_{j^{\prime} \in \mathcal{C}_{h}} w_{h j^{\prime}} q_{j^{\prime}}}, \quad j=1, \ldots, k .
$$

From (4.1) we obtain that the true probabilities $q_{t r j}, j=1, \ldots, k$ satisfy equations (7.15). Hence, $\mathbf{q}_{t r k}$ is the unique stationary point of the limit log likelihood where

$$
\max _{\mathbf{q}_{k} \in \mathcal{S}_{k-1}\left(\mathbf{q}_{t r k}\right)} 1 \mathrm{lik}{ }^{(\infty)}\left[\mathbf{q}_{k}\right]=\operatorname{llik}^{(\infty)}\left[\mathbf{q}_{t r k}\right]
$$

Corollary 7.3 Estimates $\check{\mathbf{q}}_{k M}=\check{\mathbf{q}}_{k M}\left(n_{2 \cdot}\right)$ are strongly consistent estimators of $\mathbf{q}_{t r k}$ as $n_{.2}$ is unboundedly growing.

Proof. The concave functions (4.7) uniformly converge on the compact set $S_{k-1}\left(\mathbf{q}_{t r k}\right)$ to the limit log likelihood (7.14) which has negative second order derivatives at $\mathbf{q}_{t r k}$ as $n_{.2} \rightarrow \infty$. Then it follows from (7.13) and (7.16) that the sequence of stationary points $\check{\mathbf{q}}_{k M}\left(n_{\cdot 2}\right) \rightarrow \mathbf{q}_{t r k}$.

Corollary 7.2 and 7.3 justify the possibility to use the sequence of $\mathbf{q}_{k}^{(r)}, r=$ $1,2,3, \ldots$, obtained by recursion (7.9), as consistent estimates of $\mathbf{q}_{t r k}$.

Let us show that the regularity properties RP1 - RP5 stated in Section 4 are valid. Let as in Section $4\left\{H_{i}, J_{i}\right\}, i=1, \ldots, n_{\text {.2 }}$, be independent pairs of 
r.v.s: $H_{i}=h$ if the $i$ th respondent stated interval $\mathbf{u}_{h} \in \mathcal{U}_{m}$, and $J_{i}=j$ if its WTP-point $X_{i}=x_{i} \in \mathbf{v}_{j} \in \mathcal{C}_{h}$. Let $\tilde{\mathbf{q}}_{k}=\left\{q_{1}, \ldots, q_{k-1}, q_{k}\right\}, q_{k}=1-\sum_{j=1}^{k-1} q_{j}$. We introduce the following r.v.s

$$
\mathrm{LL}_{i s}\left[\tilde{\mathbf{q}}_{k}\right]=\frac{1}{n_{\cdot 2}} \log \left[\sum_{j \in \mathcal{C}_{H_{i}}} w_{H_{i} j} q_{j}\right] \quad \text { if } \mathbf{z}_{i}=\left\{i, \mathbf{u}_{H_{i}}, N A\right\}
$$

and

$$
\mathrm{LL}_{i p}\left[\tilde{\mathbf{q}}_{k}\right]=\frac{1}{n_{\cdot 2}} \log \left[w_{H_{i} J_{i}} q_{J_{i}}\right] \text { if } \mathbf{z}_{i}=\left\{i, \mathbf{u}_{H_{i}}, \mathbf{v}_{J_{i}}\right\}
$$

$i=1, \ldots, n_{\cdot 2}, \tilde{\mathbf{q}}_{k} \in \mathcal{S}_{k-1}\left(\mathbf{q}_{t r k}\right)$. Then the normed log likelihood (4.7) can be considered as the observed value of the sum of values of r.v.s (7.17) and (7.18).

$$
\begin{aligned}
\operatorname{LLik}\left[\tilde{\mathbf{q}}_{k} \mid \hat{\mathbb{W}}_{m k}, \mathbf{d} \mathbf{2}_{n \cdot 2}\right] & =\frac{1}{n \cdot 2} \sum_{i=1}^{n \cdot 2}\left(\operatorname{LL}_{i s}\left[\tilde{\mathbf{q}}_{k}\right] I\left[\mathbf{Z}_{i}=\left\{i, \mathbf{u}_{H_{i}}, N A\right\}\right]\right. \\
& \left.+\operatorname{LL}_{i p}\left[\tilde{\mathbf{q}}_{k}\right] I\left[\mathbf{Z}_{i}=\left\{i, \mathbf{u}_{H_{i}}, \mathbf{v}_{J_{i}}\right\}\right]\right) .
\end{aligned}
$$

The $Q_{k-1}\left(\mathbf{q}_{t r k}\right)=\left\{\mathbf{q}_{k-1}=\left\{q_{1}, \ldots, q_{k-1}\right\}: \tilde{\mathbf{q}}_{k}=\left\{q_{1}, \ldots, q_{k-1}, 1-q_{1}-\cdots-\right.\right.$ $\left.\left.q_{k-1}\right\} \in \mathcal{S}_{k-1}\left(\mathbf{q}_{t r k}\right)\right\}$ is a compact set in $\mathbb{R}^{k-1}$. We denote by $\mathcal{C}_{h} \backslash k$ the exclusion of index $k$ from the set $\mathcal{C}_{h}=\left\{j: \mathbf{v}_{j} \subseteq \mathbf{u}_{h}\right\}$. For each $h=1, \ldots, m$, $\max _{j \in \mathfrak{e}_{h}} w_{h j}>0$, because if for some $h$ all $w_{h j}=0, j \in \mathfrak{C}_{h}$, then $w_{h}=0$ and the probability of $\mathbf{u}_{h}$ is zero. Then it would contradict to the observed $\mathbf{u}_{h}$ on the first step of data collection. Hence, for each $h=1, \ldots, m$ and each $\tilde{\mathbf{q}}_{k} \in$ $\mathcal{S}_{k-1}\left(\mathbf{q}_{t r k}\right)$ we have $\sum_{j \in \mathfrak{e}_{h}} w_{h j} q_{j}>0$. Then $\min _{\tilde{\mathbf{q}}_{k} \in \mathcal{S}_{k-1}\left(\mathbf{q}_{t r k}\right)} \sum_{j \in \mathfrak{e}_{h}} w_{h j} q_{j}>0$.

The first and second order partial derivatives, of the terms of log likelihood (7.19) corresponding to singles and pairs, are the following bounded and continuous functions on $\mathcal{S}_{k-1}\left(\mathbf{q}_{t r k}\right)$

$$
\frac{\partial \mathrm{LL}_{i s}\left[\tilde{\mathbf{q}}_{k}\right]}{\partial q_{j_{1}}}=\frac{w_{H_{i} j_{1}} I\left[j_{1} \in \mathcal{C}_{H_{i}} \backslash k\right]-w_{H_{i} k} I\left[k \in \mathcal{C}_{H_{i}}\right]}{\sum_{j \in \mathcal{C}_{H_{i}}} w_{H_{i} j} q_{j}},
$$

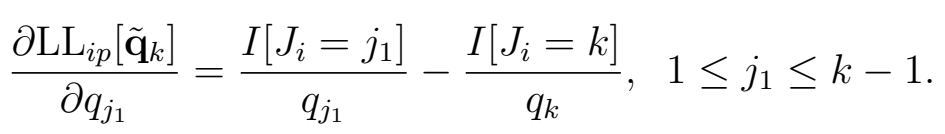


From (7.20) and (7.21) it follows that 1

$$
\begin{aligned}
\frac{\partial^{2} \mathrm{LL}_{i s}\left[\tilde{\mathbf{q}}_{k}\right]}{\partial q_{j_{1}} \partial q_{j_{2}}} & =-\left(\frac{\partial \mathrm{LL}_{i s}\left[\tilde{\mathbf{q}}_{k}\right]}{\partial q_{j_{1}}}\right)\left(\frac{\partial \mathrm{LL}_{i s}\left[\partial \tilde{\mathbf{q}}_{k}\right]}{q_{j_{2}}}\right), \quad 1 \leq j_{1}, j_{2} \leq k-1, \\
\frac{\partial^{2} \mathrm{LL}_{i p}\left[\tilde{\mathbf{q}}_{k}\right]}{\partial q_{j_{1}} \partial q_{j_{2}}} & =-\left(\frac{I\left[J_{i}=j_{1} \in \mathcal{C}_{H_{i}}\right]}{q_{j}^{2}} I\left[j_{1}=j_{2}\right]+\frac{I\left[J_{i}=k \in \mathcal{C}_{H_{i}}\right]}{q_{k}^{2}}\right) \\
& =-\frac{\partial \mathrm{LL}_{i p}\left[\tilde{\mathbf{q}}_{k}\right]}{\partial q_{j_{1}}} \frac{\partial \mathrm{LL}_{i p}\left[\tilde{\mathbf{q}}_{k}\right]}{\partial q_{j_{2}}} 1 \leq j_{1}, j_{2} \leq k-1 .
\end{aligned}
$$

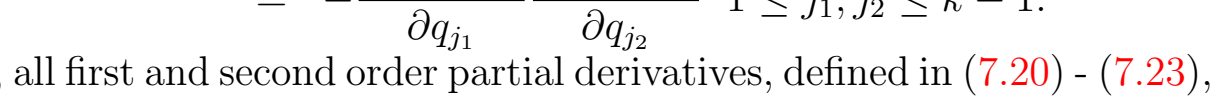

Hence, all first and second order partial derivatives, defined in (7.20) - (7.23),
are continuous and bounded functions, and the regularity property RP1 holds.

On $Q_{k-1}\left(\mathbf{q}_{t r k}\right)$ the maxima of the partial derivatives (7.20) - (7.23) are finite numbers. Hence, the above derivatives possess finite moments of any order. Therefore; the regularity property RP2 holds. From boundedness, uniform continuity of all second order partial derivatives on $Q_{k-1}\left(\mathbf{q}_{t r k}\right)$ and from the limit Lebesque theorem it follows that for any inner point $\mathbf{q}_{k-1} \in$ $Q_{k-1}\left(\mathbf{q}_{t r k}\right)$

$$
E_{\mathbf{q}_{k-1}}\left[\varlimsup_{\tilde{\operatorname{qim}}_{\tilde{\mathbf{q}}_{k-1}^{\prime} \rightarrow \tilde{\mathbf{q}}_{k}}}\left|\frac{\partial^{2} \mathrm{LL}_{i g}\left[\tilde{\mathbf{q}}_{k}^{\prime}\right]}{\partial q_{j_{1}} \partial q_{j_{2}}}-\frac{\partial^{2} \mathrm{LL}_{i g}\left[\tilde{\mathbf{q}}_{k}\right]}{\partial q_{j_{1}} \partial q_{j_{2}}}\right|>0\right]=0
$$

$g=s, p, 1 \leq j_{1}, j_{2} \leq k-1$. Relation (7.24) corresponds to the regularity property RP3. To check the regularity property $\mathbf{R P 4}$ we may use a reversible method. From relation $\sum_{h=1}^{m} \sum_{j \in \mathfrak{C}_{h}} w_{h j} q_{j}=1$, (7.18) and (7.20) we have

$$
\begin{aligned}
0 & =\frac{1}{n_{\cdot 2}} \frac{\partial}{\partial q_{j_{1}}} \sum_{h=1}^{m} \sum_{j \in \mathfrak{C}_{h}} w_{h j} q_{j} \\
& =\frac{1}{n_{\cdot 2}} \sum_{h=1}^{m}\left(\frac{w_{h j_{1}}-w_{h k} I\left[k \in \mathcal{C}_{h}\right]}{\sum_{j \in \mathfrak{C}_{h}} w_{h j} q_{j}}\right) \sum_{j \in \mathfrak{C}_{h}} w_{h j} q_{j} \\
& =\frac{1}{n_{\cdot 2}} \sum_{h=1}^{m} \frac{\partial}{\partial q_{j_{1}}} \log \left[\sum_{j \in \mathfrak{C}_{h}} w_{h j} q_{j}\right] \sum_{j \in \mathfrak{C}_{h}} w_{h j} q_{j}=E_{\tilde{\mathbf{q}}_{k}}\left[\frac{\partial \mathrm{LL}_{i s}\left[\mathbf{q}_{k}\right]}{\partial q_{j_{1}}}\right]
\end{aligned}
$$

$1 \leq j_{1} \leq k-1$. One may read (7.25) from right to left. Similarly we obtain

$$
E_{\tilde{\mathbf{q}}_{k}}\left[\frac{\partial \mathrm{LL}_{i p}\left[\mathbf{q}_{k}\right]}{\partial q_{j_{1}}}\right]=0, \quad 1 \leq j_{1} \leq k-1
$$


From (7.22) and (7.23) we obtain

$$
E_{\tilde{\mathbf{q}}_{k}}\left[\frac{\partial^{2} \mathrm{LL}_{i g}\left[\mathbf{q}_{k}\right]}{\partial q_{j_{1}} \partial q_{j_{2}}}\right]=-E_{\tilde{\mathbf{q}}_{k}}\left[\frac{\partial \mathrm{LL}_{i s}\left[\mathbf{q}_{k}\right]}{\partial q_{j_{1}}} \frac{\partial \mathrm{LL}_{i s}\left[\mathbf{q}_{k}\right]}{\partial q_{j_{2}}}\right], \quad g=s, p .
$$

2

Validity of (7.25) - (7.27) means that the regularity property $\mathbf{R P} \mathbf{4}$ holds.

Let us introduce two $(k-1) \times(k-1)$ matrices

$$
\mathbb{F}_{s n \cdot 2}\left[\mathbf{q}_{k-1}\right]=-\frac{n_{s 2}}{n_{\cdot 2}}\left(E_{\mathbf{q}_{k-1}}\left[\frac{\partial^{2} \mathrm{LL}_{1 s}\left[\mathbf{q}_{k-1}\right]}{\partial q_{j_{1}} \partial q_{j_{2}}}\right]\right)_{1 \leq j_{1}, j_{2} \leq k-1}
$$

$$
\mathbb{F}_{p n \cdot 2}\left[\mathbf{q}_{k-1}\right]=-\frac{n_{p 2}}{n_{\cdot 2}}\left(E_{\mathbf{q}_{k-1}}\left[\frac{\partial^{2} \mathrm{LL}_{1 p}\left[\mathbf{q}_{k-1}\right]}{\partial q_{j_{1}} \partial q_{j_{2}}}\right]\right)_{1 \leq j_{1}, j_{2} \leq k-1}
$$

The Fisher Information (FI-)matrix corresponding the log likelihood (4.7) is

$$
\mathbb{F}_{n \cdot 2}\left[\mathbf{q}_{k-1}\right]=\mathbb{F}_{s n \cdot 2}\left[\mathbf{q}_{k-1}\right]+\mathbb{F}_{p n \cdot 2}\left[\mathbf{q}_{k-1}\right], \quad \mathbf{q} \in Q_{k-1}\left(\mathbf{q}_{t r k}\right)
$$

From (7.27) - (7.29) it follows that matrix $\mathbb{F}_{\text {sn.2 }}\left[\mathbf{q}_{k-1}\right]$ is non-negative and $\mathbb{F}_{p n \cdot 2}\left[\mathbf{q}_{k-1}\right]$ is positive definite with full rank $(n-1)$. Then defined in (7.30) the FI-matrix $F_{n \cdot 2}\left[\mathbf{q}_{k_{1}}\right]$ has full rank. Hence the regularity property RP5 also holds.

We summarize obtained results in the following

THEOREM 7.2 The regularly properties RP1 - RP5 are valid for the statistical model with the log likelihood (4.7).

Let $\mathfrak{M}=\{\mathcal{M}\}$ be a class of statistical models $\mathcal{M}$ which possess the following properties:

PD: the set of data in the model $\mathcal{M}$ is a list $\mathbf{d}_{n}=\left\{\mathbf{x}_{1}, \ldots, \mathbf{x}_{n}\right\}$ which components, $\mathbf{x}_{i} \in \mathbb{R}^{d}$, are values of independent, r.v.s, and their distributions can belong to different families of parametric distributions however with the same set $\Theta=\{\boldsymbol{\theta}\} \subseteq \mathbb{R}^{k}$ of parameters, 
PE: there exists a consistent ML-estimate $\check{\boldsymbol{\theta}}_{n}$ of the true parameter $\boldsymbol{\theta}_{0} \in$ $\Theta$;

PR: the $\log$ likelihoods of components $\mathbf{x}_{i} \in \mathbf{d}_{n}$ possess the standard regularity properties, (similar with PR1 - PR5 stated in Section 4 and checked above in this Appendix.)

Proposition 1 Let the properties $\mathbf{P D}, \mathbf{P E}, \mathbf{P R}$ be valid for a model $\mathcal{M} \in \mathfrak{M}$. Then the distribution of normed deviations $\sqrt{n}\left(\check{\theta}_{n}-\boldsymbol{\theta}_{0}\right)$ approaches, as $n \rightarrow \infty$, with d-dimensional Normal distribution with the covariance matrix equal to the inverse FI-matrix.

This result is a convenient slight generalisation of a well known classical result of R. Fisher, see Lehmann and Casella (1998), Ferguson (1996).

Let us $\mathbf{d}_{n}^{\star c}=\left\{\mathbf{X}_{\tilde{i}_{1}^{\star c}}, \ldots, \mathbf{X}_{\tilde{i}_{r}^{\star c}}\right\}$ be resampled copy of the original data $\mathbf{d}_{n}$. Then we can restate properties $\mathbf{P D}, \mathbf{P E}$ and $\mathbf{P R}$ by using the resampled copies of data $\mathbf{d}_{n}^{\star c}$ instead of $\mathbf{d}_{n}$. We denote these properties by $\mathbf{P} \mathbf{D}^{\star \mathbf{c}}, \mathbf{P} \mathbf{E}^{\star c}$ and $\mathbf{P R}^{\star c}$. For example, we have

$\mathbf{P E}^{\star c}$ : there exits a consistent ML-estimate $\check{\boldsymbol{\theta}}_{n}^{\star c}$ of the true parameter $\boldsymbol{\theta}_{0} \in \Theta$.

Proposition 2 Let the properties $\mathbf{P D}, \mathbf{P E}, \mathbf{P R}$ and $\mathbf{P D} \mathbf{D}^{\star \mathbf{c}}, \mathbf{P E}^{\star c}, \mathbf{P R}^{\star c}$ be valid for a model $\mathcal{M} \in \mathfrak{M}$. Then the distributions of $\sqrt{n}\left(\check{\boldsymbol{\theta}}_{n}-\boldsymbol{\theta}_{0}\right)$ and $\sqrt{n}\left(\check{\boldsymbol{\theta}}_{n}^{\star c}-\check{\boldsymbol{\theta}}_{n}\right)$ are approaching each other in probability as $n \rightarrow \infty$.

The Proposition 2 is valid for the considered statistical model with twostep sampling design with self-selected rounded interval data.

The Proposition 1 and 2 are proved in Belyaev and Nilsson (1997), see there Theorem 5 and 6, in Nilsson (1998) there are also several applications and detailed proofs. The asymptotic properties of resapled sums of independent r.v.s are considered in Belyaev and Sjöstedt-de Luna (2000); Belyaev (2003, 2007). 


\section{REFERENCES}

Belyaev, Y. (2003): Necessary and sufficient conditions for consistency of resampling. Research report, Center of Biostochastics. Swedish University of Agricultutal Sciences. http://biostochastics.slu.se/publikationer.

Belyaev, Y. (2007): Resampling for Lifetime Distributions. In Encyclopedia of Statistics in Quality and Reliability, Ed. Rupper, F., Kennet, R., Faltin, F.W. John Wiley, Chichester, UK, 1653-1657.

Belyaev, Y. And Kriström, B. (2010): Approach to Analysis of Self-selected Interval Data. CERE Working Paper \#2/2010.

Belyaev, Y. And Kriström, B. (2012): Analysis of contingent valuation data with selfselected ounded WTP-intervals collected by two-steps sampling plans. Proceedings of the $9^{\text {th }}$ Conference on Multivariate Statistics. Tartu, 2011. (In print).

Belyaev, Y. And Nilsson, L. (1997): Parametric Maximum Likelohood Estimators and Resampling. Research report, N15. Department of Mathematical Statistics.

Belyaev, Y. And Sjöstedt-de Luna, S. (2000): Weakly approaching sequences of random distributions. J. Applied Probability, 37, 807-822.

Brinkhuis, J. And Tihomirov, V. (2005): Optimization: Insights and Applications. Princeton University Press, Princeton and Oxford.

Broberg, T. AND BränNlund, R. (2008): An alternative interpretation of multiple bounded WTP data-Certainty dependent payment card intervals. Resources Energy Econ, doi:10.1016/j.reseneeco.2008.09.001.

Davison, A.C. And Hinkley, D. (1997): Bootstrap Methods and their Applications. Cambridge University Press. Cambridge.

Ferguson, T.S. (1996): A Course in Large Sample Theory. Chapman \& Hall, London. FInk, A. (1985): How to ask survey questions. Thousand Oaks, CA: SAGE Publications. Wiley, New York.

Garthwaite, P.H., Kadane, J.B. and A O'Hagan (2004): "Elicitation" Technical Report 04/01, 2004, Open University. http://statistics.open.ac.uk/ TechnicalReports/Handbook-Elicitation.pdf

HobBs, F. (2004): Age and Sex Composition, in Siegel, B. and Swansson, D. (eds), The methods and Materials of demography, Elserier Academic Press, San Diego, CA, USA.

Hurd, M.D., McFadden, D., Chand, H., Gan, L., Merrill, A. and Roberts, M. (1998): Consumption and savings balances of the elderly: Experimental evidence on 
survey response bias, in D. Wise (Ed.) Frontiers in the Economics of Aging, 353-387, Chicago, IL: University of Chicago Press.

2

Huttenlocher, J., Hedges, L.V. And Bradburn, N.M. (2008): Reports of elapsed time: Bounding and rounding processes in estimation.Journal of Experimental Psychology: Learning, Memory, and Cognition 16, 196-213.

HÅkansson, C. (2007): Cost-Benefit Analysis and Valuation Uncertainty. Doctoral Thesis, No. 2007-41, Swedish University of Agricultural Sciences, Umeå.

HÅKANSSON, C. (2008): A new valuation question - Analysis of and insights from interval open ended data in contingent valuation. Environmental and Resource Economics 39,2,175-188.

Jammalamadaka, S.R. ANd Mangalam, V. (2003): Non-parametric estimation for middle-censored data. J. Nonparametr. Statist. 15, 253-265.

Klein, J.P. And Moeschberger, ML (1997): Survival Analysis: Techniques for Censored and Truncated Data, Springer-Verlag.

Lehmann, E.L. And Casella, G. (1998): Theory of point estimation. 2nd. ed., N. Y.: Springer-Verlag.

Manski, C.F. and F.Molinari (2010) Rounding Probabilistic Expectations in Surveys. Journal of Business and Economic Statistics. 28, 219-231.

McFadden, D., Bemmaor, A., Caro, F., Dominitz, J., Jun, B-H., Lewbel, A., Matzkin, R., Molinari, F., Schwarz, N., Willis, R. and Winter, J. (2005): Statistical Analysis of Choice Experiments and Surveys, Marketing Letters, 16, 183196.

Nilsson, L. (1998): Parametric Estimation Using Computer Intensive Methods. Ph. Dissertation, ISBN 91-7191-438-8, Dept. of Mathematical Statistics, Umeå University. Rosch, E. (1975): Cognitive reference points. Cognitive Psychology 7, 532-547.

Schaeffer, N.C. And Bradburn, N.M. (1989): Respondent behaviour in magnitude estimation. Journal of the American Statistical Association 84, 402-413.

Turnbull, B.W. (1976): The empirical distribution function with arbitrarily grouped, censored and truncated data. J. Roy. Statist. Soc. Ser. B 38, pp. 290-295.

van Exel, W.B.F., Brouwer, B., van den Berg, B. and Koopmanschap, M.A. (2006): With a little help from an anchor discussion and evidence of anchoring effects in contingent valuation, The Journal of Socio-Economics, 35, 836-853.

Wenchao, Z., Belyaev, Y. And Kriström, B. (2012): Package Software for Analysis of Rounded Self-Selected Interval Data (PSARSSID). Swedish University of Agricultutal Sciences. (In preparation). http://www.cere.se . 